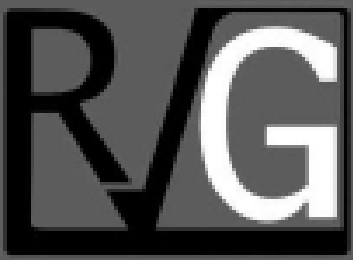

Año 24 No. 88

Octubre - Diciembre 2019

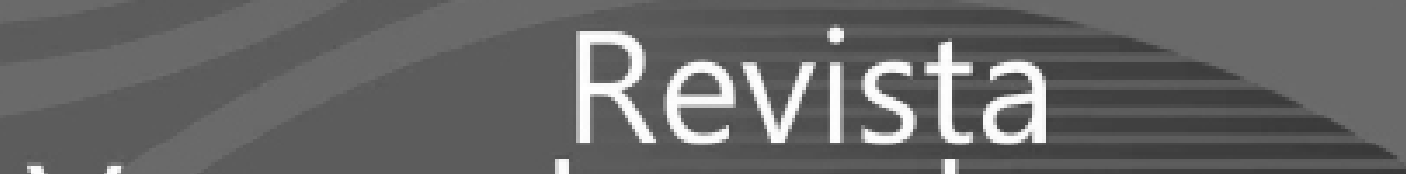

Venezolana de

verencla

UNIVERSIDAD DEL ZULIA (LUZ)

Facultad de Ciencias Económicas y Sociales

Centro de Estudios de la Empresa 


\title{
Emprendimiento con pertinencia social y territorial. Caso: Universidad Católica de Cuenca, Azogues- Ecuador ${ }^{1}$
}

\author{
Solis Muñoz, Juan Bautista ${ }^{2}$ \\ García Alvarez, Florencio Iván ${ }^{3}$ \\ Mantilla Crespo, Xaxier Augusto ${ }^{4}$
}

\section{Resumen}

Uno de los problemas de la planificación del Ecuador, es la débil articulación academia- industria y sociedad. La investigación describe el emprendimiento y la innovación con pertinencia social y territorial, mediante fundamentación teórica de las variables de interés y resultados empíricos encontrados. El emprendimiento e innovación es la variable dependiente y las variables predictoras: la pertinencia social y territorial; y, emprendimientos interculturales innovadores desde el diálogo de saberes. Se trabaja sobre 70 proyectos de emprendimiento, a nivel de modelos de negocios, en la carrera de Ingeniería Empresarial de la Universidad Católica de Cuenca, Sede Azogues. Los indicadores de la tasa mínima aceptable de retorno, valor neto actual y tasa interna de retorno, en la mayoría de los rangos, son favorables a la viabilidad económica y financiera de los emprendimientos. Pero, tan solo el 3\% de los emprendimientos son implementados, tasa que afecta sensiblemente a la pertinencia social de los emprendimientos. Se debe enriquecer y potenciar la línea de investigación institucional y los ámbitos estratégicos de intervención mediante innovación radical que oriente la pertinencia social de la oferta académica a la generación de riqueza y empleo.

Palabras clave: Emprendimiento; innovación; pertinencia social y territorial; diálogo de saberes e interculturalidad; Ecuador.

\section{Recibido: 27-05-19 Aceptado: 25-09-19}

$1 \quad$ Esta investigación presenta resultados parciales del estudio de pertinencia de la carrera de Administración de Empresas de la Universidad Católica de Cuenca Sede Azogues, con base en los trabajos de titulación en el periodo 2012- 2018 en pregrado.

2 Universidad Católica de Cuenca Sede Azogues, Carrera de Administración de Empresas. Azogues, Ecuador. jbsolizm@ucacue.edu.ec

3 Universidad Católica de Cuenca Sede Azogues, Carrera de Administración de Empresas. Azogues, Ecuador figarciaa@ucacue.edu.ec

$4 \quad$ Universidad Católica de Cuenca Sede Azogues, Carrera de Administración de Empresas. Azogues, Ecuador. xamantillac@ucacue.edu.ec 


\title{
Entrepreneurship with social and territorial relevance. Case: Universidad Católica de Cuenca branch Azogues Ecuador
}

\begin{abstract}
One of the problems of Ecuador's planning is the weak between academia-industry and society articulation. This research describes entrepreneurship and innovation with social and territorial relevance, to achieve the description, the theoretical foundation of the variables of interest was used and empirical results found, too. In this sense, entrepreneurship and innovation is the dependent variable and the predictive variables are social and territorial relevance as well as pioneering intercultural entrepreneurship from the dialogue of knowledge. It has been working on 70 entrepreneurship projects at the level of business models, in Business Engineering Major at Universidad Católica de Cuenca branch Azogues. The indicators of the minimum acceptable rate of return, current net value and internal rate of return in most of the ranges are favorable to the economic and financial feasibility of the enterprises. However, only $3 \%$ of the entrepreneurships are implemented, a rate that significantly affects the social relevance of the enterprises. The line of academic institutional research and strategic areas of intervention must be enriched and strengthened through drastic innovation that guides the social relevance of the study field to the generation of wealth and employment.
\end{abstract}

Key Works: Entrepreneurship; innovation; social and territorial relevance; dialogue of knowledge and interculturality; Ecuador.

\section{Introducción}

La necesidad de fomento del emprendimiento e innovación toma fuerza en Ecuador, desde los incentivos previstos en la Ley Orgánica para el Fomento Productivo, Atracción de Inversiones, Generación de Empleo, Estabilidad y Equilibrio Fiscal que promueve el dinamismo económico mediante la inversión y el empleo que garantice la sostenibilidad fiscal a largo plazo bajo un esquema de certidumbre y seguridad jurídica como mecanismo que impulsa la productividad y la competitividad de los sectores económicos del país. En esta línea, el rol de la Universidad es determinante en la formación de profesionales emprendedores e innovadores y el acompañamiento a los emprendedores autónomos a través de la implementación de un modelo de incubación de empresas.

Para ello, la carrera de Ingeniería Empresarial de la Universidad Católica de Cuenca Sede Azogues, requiere conocer los resultados de los modelos de negocios sustentados por los estudiantes de grado y el posible impacto en la actividad económica de los cantones de Azogues, Biblián y Déleg, área de influencia de la institución de educación superior. Los hallazgos servirán a la 
función directiva para adoptar decisiones curriculares y administrativas con base en la pertinencia social y territorial de la Carrera, orientadas al aprovechamiento de las oportunidades de entorno.

Con el antecedente se busca el fortalecimiento de la articulación público - privada y academia- emprendimiento, situado en el Plan Nacional de Desarrollo, Toda una Vida (Secretaria Nacional de Planificación y Desarrollo, 2017: 72) como un problema a resolver en las agendas territoriales. En efecto, en el Eje 2 de la planificación nacional: Economía al servicio de la sociedad, en concordancia con el objetivo 5, se precisa, "Impulsar la productividad y competitividad para el crecimiento económico sostenible, de manera redistributiva y solidaria", lo que configura un punto de sustento para el emprendimiento e innovación en el Ecuador.

En la línea del Plan nacional de Desarrollo, Toda una Vida, se establecen los marcos de acción encaminadas a operacionalizar el propósito al "promover la productividad, competitividad y calidad de los productos nacionales (...) para generar valor agregado y procesos de industrialización en los sectores productivos con enfoque a satisfacer la demanda nacional y la exportación". De igual forma, las políticas también se orientan a "promover la investigación, la formación, la capacitación, el desarrollo y la transferencia tecnológica, la innovación y el emprendimiento (...) para impulsar el cambio de la matriz productiva mediante la vinculación entre el sector público, productivo y las universidades" ((Secretaria Nacional de Planificación y Desarrollo, 2017:74).

Desde el antecedente, siguiendo los lineamientos del Plan Nacional de Desarrollo, Toda una Vida (2017) una de las metas que aterriza la pertinencia territorial de los actores de la economía popular y solidaria es "implementar modelos estratégicos de negocios que permitan la planificación del desarrollo de la organización" (Secretaria Nacional de Planificación y Desarrollo, 2017:75). Lo dicho está corroborado por lo dispuesto en el artículo 107 de la Ley Orgánica de Educación Superior, reformado que al referirse a la necesidad de articular a la universidad con la sociedad expresa: (Presidencia de la Republica, 2018).

El principio de pertinencia consiste en que la educación superior responda a las expectativas y necesidades de la sociedad, a la planificación nacional, y al régimen de desarrollo, a la prospectiva de desarrollo científico (...) Por ello, las instituciones de educación superior articularán su oferta docente de investigación y actividades de vinculación con la sociedad, a la demanda académica, a las necesidades de desarrollo local, regional y nacional, a la innovación y diversificación de profesionales y grados académicos, a las tendencias del mercado ocupacional local, regional y nacional (Secretaria Nacional de Planificación y Desarrollo, 2017:18)

Se toma en cuenta que el Ecuador se encuentra regionalizada por zonas de planificación, la Universidad Católica de Cuenca Sede Azogues, se encuentra ubicada en la Zona de Planificación 6 en la que se desarrollan los campos de estudio y actuación de la profesión de Ingeniería Empresarial tomando en cuenta el objetivo específico centrado en el diseño de proyectos que tributan al desarrollo zonal. Por tanto, los Dominios Académicos de la Universidad Católica de Cuenca (2016:1) determinan el "Desarrollo empresarial sostenible en grandes, medianas y pequeñas empresas" en correspondencia con las líneas y de investigación: "Gestión 
empresarial con responsabilidad social" y los ámbitos de acción estratégicos: "Creación de Empresas y gestión de PYMES, tecnologías avanzadas de gestión empresarial y modelado, organización y estrategia empresarial, emprendimiento estratégico" (Secretaria Nacional de Planificación y Desarrollo, 2017:1).

El artículo presenta las tensiones y reflexiones, a partir de la revisión sistemática de literatura con relación al emprendimiento e innovación y las categorías básicas de: Pertinencia social y territorial; Emprendimientos interculturales innovadores desde el diálogo de saberes; e, Impacto social, económico y cultural del emprendimiento e innovación. La sustentación teórica que antecede inserta la necesidad de convergencia entre el conocimiento científico y el conocimiento cotidiano para emerger propuestas de emprendimientos con base en la inteligencia colectiva y el principio de sostenibilidad.

En concordancia con el objetivo planteado, la investigación se sitúa en el paradigma positivista. Se empleó el enfoque de indagación cuantitativa, descriptiva, de corte no experimental. Se partió desde la revisión sistemática de literatura pertinente al emprendimiento y liderazgo que pesan como variable dependiente. En forma similar se rastreó las miradas y tensiones teóricas alrededor de las variables predictoras: Pertinencia social y territorial; $\mathrm{y}$, emprendimientos interculturales innovadores desde el diálogo de saberes. Las novedades teóricas permitieron construir los indicadores que se presentan en el apartado de hallazgos. Para ello, se usó las bases de datos de trabajos de titulación de la carrera de Ingeniería Empresarial de la Universidad Católica de Cuenca Sede Azogues, en el periodo
2012-2018 con 70 casos de diseño de modelos de negocios que arrojó datos de interés para las variables de estudio, lo que permitió la construcción de una posición teórico-empírica sobre la pertinencia y relevancia de la línea y los ámbitos de investigación institucionales, a partir del músculo teórico que corrobora los hallazgos cuantitativos.

\section{Reflexiones en torno al emprendimiento y la innovación}

Al aludir al emprendimiento, se recurre a lo planteado por Schumpeter (1984), quien define al emprendedor como persona con ideas creativas dispuestas a correr riesgos en escenarios de incertidumbre para transformar la realidad social, económica y cultural en un contexto específico desarrollando las competencias y habilidades laborales para responder asertivamente al segmento de mercado objetivo.

Para ello, la enseñanza del emprendimiento, constituye un eje transversal (Castillo, 1999), cuyas líneas teóricas y modelos se generan a partir de los cursos de pre y pos grado y también el personal de nivel ejecutivo y emprendedores. Para cumplir con las expectativas de formación se recurre a la experiencia de emprendedores exitosos quienes son convocados para contar historias que contextualizan los desafíos y empeños del emprendimiento social e inclusivo.

En consecuencia, el emprendimiento se define como el crecimiento sostenido de un plan multicriterio, dotado de factores específicos en escenarios dinámicos que apuntan a la innovación del producto y de la gestión empresarial (Formichella, 
2004). Tomando en cuenta también la apreciación que sobre empresas Pyme o empresarios Pyme hace Vicente y Meleán (2018) al indicar que es adecuado en todo caso tratar el tema bajo la etiqueta de empresario Pyme en vez de empresa Pyme en razón que la pequeña y mediana empresa ve la luz desde la acción de sus fuerzas inmanentes de oferta y demanda, teniendo de por medio al emprendedor como su pilar y fundamento.

En este sentido, desde la línea conceptual de emprendimiento, se hace necesario comprender la prospectiva del emprendimiento empresarial en opinión de Rodríguez (2009) se trata de una orientación a la toma de decisiones para el aprovechamiento óptimo de las externalidades positivas del entorno con base en sus competencias y dominios, creatividad para transformar productos $y$ procesos a través de la innovación.

Por otro lado, en lo que compete a la innovación se define desde distintas visiones, contextos y momento históricos. Así en la línea de pensamiento de Schumpeter (1934) se trata de un estado de implosión o destrucción creativa que da paso a nuevos productos y procesos. Por su parte, Gee (1981) sostiene que la innovación se cimenta sobre engranajes nacidas de las ideas promovidas por sensaciones de carencia unidas al deseo de hacerlas desaparecer al converger en productos que son bienes y servicios ofrecidos al mercado. Para Pavón y Goodman (1981) en cambio, innovar es la generación de un impacto positivo y original de un nuevo producto que agrega valor para el consumidor cliente.

En la línea de pensamiento de Nelson y Winter (1982) la innovación es sinónimo de ruptura profunda entre un estado histórico y un escenario deseado.
Fortalece el criterio del salto cuántico al asociar lo que actualmente se le conocer como la revolución industrial 4.0 a la innovación en el componente de la digitalización (Perrin, 1995). Corrobora lo dicho Machado (1997) cuando afirma que la innovación tiene un componente tecnológico que da paso a un momento de cambio que incide en la productividad del proceso y la competitividad de la gestión empresarial. Por el mismo estadio de tiempo Pavón e Hidalgo (1997) sostiene que la innovación tiene resonancia en los nichos de transformación de insumos en productos terminados y finales listos para ser comercializados, es decir, el espacio es netamente técnico.

Desde los esfuerzos de la comunidad global por aprovechar los beneficios de la innovación, el Manual de Oslo (2018) incorpora a la innovación de producto también la innovación de la gestión empresarial y amplía el concepto de innovación argumentando que su alcance es a una actividad o al resultado de una actividad. Lo dicho, da pie al impulso de emprendimiento innovadores en el campo de la gestión de negocios.

La pertinencia social y territorial desde la mirada de Zafra, Martínez y Vergel (2014) es una categoría histórica, espacial y prospectiva. Implica un punto donde convergen los recursos y los esfuerzos personales e institucionales con el propósito de transformar la realidad social, económica y cultural de un sector geográfico, donde residen etnias y pueblos ancestrales como también corrientes modernas de asentamientos humanos.

Así, en el contexto centroamericano, Nicaragua ha desarrollado la producción agropecuaria a gran escala, tributando al crecimiento del país. Sin embargo, la pertinencia social y territorial sostenida, tiene serios 
cuestionamientos por la brecha de desigualdades sociales y económicas que ha crecido exponencialmente debido a la concentración de los factores de la producción en pocas manos (Grisgby, 2010).

Desde esta lógica, el repensar universitario implica creativas formas de encarar los cambios globales con identidad local y los efectos en la forma de hacer las cosas en el ámbito académico. La pertinencia social y territorial como dimensión tempo espacial re direcciona el rol universal, a partir de la necesidad de articular a la universidad con la sociedad, torna imprescindible la revisión reflexiva de planes, programas $y$ proyectos académicos (Naidorf, Giordana y Horn, 2007).

Así, los modelos de negocios que se propone a nivel de grado en las escuelas, facultades y carreras de administración de empresas deben sintonizarse con el principio de pertinencia social y territorial en sus contextos específicos. Así, la necesidad de replantear emprendimientos en función de las ventajas comparativas y competitivas de la vocación, cultural, ambiental, económica y productiva de los contextos locales, en tanto lo urbano se configura con las influencia de la ruralidad.

En consecuencia, ¿Cómo el diálogo de saberes implica mediación cultural entre el conocimiento científico y el ancestral, con pertinencia a la identidad territorial? para potenciar la interacción y convivencia de los núcleos sociales basada en los aportes teóricos multidisciplinarios, en un nivel de construcción crítica y compleja que define: 1) Diálogo de saberes como la tensión dialéctica del conocimiento científico con el originario desde la resignificación y visualización del debate intercultural, pluricultural y multiétnico. 2) La sustentabilidad y sostenibilidad como la convergencia e inmersión de la Academia en la cosmovisión andina. 3) El equilibrio alimentario como el balance producción- consumo garantizando los derechos de la Pachamama, comunas y comunidades a sistemas ecológicamente sustentables.

La investigación e innovación en la cadena de valor productiva fomenta la cooperación en un ambiente de equidad y solidaridad. El abordaje de los conceptos precedentes, permite el aseguramiento de la calidad de vida de la población y la transferencia del conocimiento intergeneracional con dignidad, para articular la propuesta académica como espacio de inter-aprendizaje dinámico, incierto $y$ en permanente prospectiva (Barbero, 2008).

Para cerrar el abordaje teórico, se inserta un caso de estudio en el contexto ecuatoriano realizado por la Facultad de Ciencias Administrativas de la Universidad Técnica de Ambato que surge desde la necesidad de las instituciones de educación superior por producir proyectos de emprendimiento con innovación. El enfoque corresponde a la relación de dominios cognitivos como prerrequisito al emprendimiento. Los hallazgos de alta incidencia determinan que los estudiantes no conocen las líneas de emprendimiento que emprende la Carrera de Administración. Los profesores universitarios tampoco impulsan la generación de proyectos viables y los contenidos académicos no se corresponden con las necesidades del sector económico. La correlación entre el emprendimiento y las competencias cognitivas tienen un coeficiente de Pearson del 0,545. Se recomienda la implementación de un sistema patrón de gestión administrativa que motive 
la generación de emprendimientos innovadores (Jiménez, Larrea, Navarrete y Castro, 2019).

En consecuencia, corresponde orientar las líneas y ámbitos de investigación de las instituciones de educación superior a soldar los eslabones de la cadena: academia, industria, estado, asumiendo la transversalidad del emprendimiento y la innovación a toda la oferta académica. Propiciando espacios de dualidad academia- industria y aprovechando las bondades de la política pública en el ámbito de la asociatividad y la transferencia del conocimiento. Los trabajas de titulación de pre y pos grado deben ser propiciadores de modelos de negocios en los sectores de la economía para crear riqueza y generar empleo productivo.

\section{Emprendimiento e} innovación con pertinencia social y territorial. Reflexiones desde el diálogo de saberes, carrera de Ingeniería

\section{Empresarial}

En este apartado se muestran los resultados de campo de 70 emprendimientos que provienen de los trabajos de titulación de grado en la carrera de Ingeniería Empresarial de la Universidad Católica de Cuenca, Sede Azogues. A partir de la metodología declarada, con base en los aportes teóricos rastreados sistemáticamente.

En la tabla 1 se muestra que, de una población de 70 emprendimientos estudiados, 14 de estos con una equivalencia relativa del $20 \%$ se ubican en el rango entre $11 \%$ y $15 \%$ como tasa mínima aceptable de retorno que asocia los factores de riesgo e inflación. Seguido por el $11,43 \%$ de emprendimientos que aspiran tasa mínima que va desde el $16 \%$ al $20 \%$ lo que implica una razonable formulación de la propuesta en escenarios de sensibilidad al precio y a la incertidumbre. Además, a nivel de pre inversión los perfiles de emprendimientos siguen la matriz metodológica y desencadenan en flujos de caja que permite la evaluación de los proyectos.

\section{Tabla 1}

Tasa mínima aceptable de retorno de los emprendimientos, periodo 2012-2018 Carrera de Ingeniería Empresarial

\begin{tabular}{ccc}
\hline Rango & $\begin{array}{c}\text { TMAR } \\
\text { Frecuencia }\end{array}$ & Porcentaje \\
\hline $0 \%-5 \%$ & 0 & $0,00 \%$ \\
$5 \%-10 \%$ & 3 & $4,29 \%$ \\
$11 \%-15 \%$ & 14 & $20,00 \%$ \\
$16 \%-20 \%$ & 8 & $11,43 \%$ \\
$21 \%-25 \%$ & 1 & $1,43 \%$ \\
$26 \%-30 \%$ & 0 & $0,00 \%$ \\
$31 \%-35 \%$ & 0 & $0,00 \%$ \\
$36 \%-40 \%$ & 1 & $1,43 \%$ \\
No dispone & 43 & $61,43 \%$ \\
\hline Total & 70 & $100 \%$ \\
\hline
\end{tabular}

Fuente: Elaboración propia a partir del trabajo de campo realizado

En lo que respecta al valor neto actual indicador que se muestra en la tabla 2 implica valores positivos establecidos en los rangos de presentación de los datos, lo que implica que los flujos futuros traídos a valor presente son favorables al proyecto 
contribuyendo a una primera certeza para movilizar recursos al proyecto en la etapa de inversión.

\begin{tabular}{|c|c|c|}
\hline \multicolumn{3}{|c|}{ VAN } \\
\hline Rango & Frecuencia & Porcentaje \\
\hline $0,00-10.000,00$ & 10 & $14 \%$ \\
\hline $10.001,00-20.000,00$ & 8 & $11 \%$ \\
\hline $20.001,00-30.000,00$ & 5 & $7 \%$ \\
\hline $30.001,00-40.000,00$ & 7 & $10 \%$ \\
\hline $40.001,00-50.000,00$ & 3 & $4 \%$ \\
\hline $50.001,00-60.000,00$ & 2 & $3 \%$ \\
\hline $60.001,00-70.000,00$ & 2 & $3 \%$ \\
\hline $70.001,00-80.000,00$ & 0 & $0 \%$ \\
\hline $80.001,00-90.000,00$ & 3 & $4 \%$ \\
\hline $90.001,00-100.000,00$ & 2 & $3 \%$ \\
\hline $100.001,00-110.000,00$ & 2 & $3 \%$ \\
\hline Más de $110.000,00$ & 16 & $23 \%$ \\
\hline No dispone & 10 & $14 \%$ \\
\hline Total & 70 & $100 \%$ \\
\hline
\end{tabular}

Fuente: Elaboración propia a partir del trabajo de campo realizado

Tabla 3

Tasa interna de retorno de los emprendimientos, periodo 2012-2018. Carrera de Ingeniería Empresarial

\begin{tabular}{ccc}
\hline Rango & $\begin{array}{c}\text { TIR } \\
\text { Frecuencia }\end{array}$ & Porcentaje \\
\hline $0 \%-5 \%$ & 1 & $1 \%$ \\
$5 \%-10 \%$ & 1 & $1 \%$ \\
$11 \%-15 \%$ & 4 & $6 \%$ \\
$16 \%-20 \%$ & 8 & $11 \%$ \\
$21 \%-25 \%$ & 12 & $17 \%$ \\
\hline
\end{tabular}

\section{Cont... Tabla 3}

\begin{tabular}{ccc}
\hline $26 \%-30 \%$ & 5 & $7 \%$ \\
$31 \%-35 \%$ & 9 & $13 \%$ \\
Más de 35\% & 20 & $29 \%$ \\
No dispone & 10 & $14 \%$ \\
\hline Total & 70 & $100 \%$ \\
\hline
\end{tabular}

Fuente: Elaboración propia a partir del trabajo de campo realizado

Los hallazgos con relación a la tasa interna de retorno, presentados en la tabla 3 hacen notar que tan solo el $2 \%$ de las tasas de los emprendimientos son menos o iguales a la tasa de descuento del $10 \%$. En adelante, las tasas de retorno son mayores a la tasa de descuento atribuyendo la decisión favorable para su implementación. Implica también un indicador decisivo para reactivar la economía en los sectores primarios, industriales, de servicios y de la economía popular y solidaria. Se genera confianza en el sector productivo y se desacelera la inversión financiera especulativa.

\section{Tabla 4}

Ubicación de los

Emprendimientos derivados del periodo 2012-2018. Carrera de Ingeniería Empresarial.

\begin{tabular}{ccc}
\hline Ubicación de los proyectos de emprendi- & $\begin{array}{c}\text { miento } \\
\text { Frecuencia }\end{array}$ & Porcentaje \\
\hline Localidad & 46 & $65,71 \%$ \\
Azogues & 7 & $10,00 \%$ \\
Biblián & 4 & $5,71 \%$ \\
Cañar & 1 & $1,43 \%$ \\
Cuenca & 3 & $4,29 \%$ \\
Javier Loyola & 1 & $1,43 \%$ \\
Nazón & 8 & $11,43 \%$ \\
No dispone & 70 & $100 \%$
\end{tabular}

Fuente: Elaboración propia a partir del trabajo de campo realizado 
En la tabla 4 se describe la pertinencia territorial de los emprendimientos tomando en cuenta el área de influencia de la carrera de Ingeniería Empresarial y la vocación económica y productiva de los cantones Azogues, Biblián y Déleg en la provincia del Cañar como parte de la zona de planificación Z6 Senplades. La distribución porcentual de los emprendimientos por cantones coherente con la línea y ámbitos de investigación y refleja el nivel de penetración de la carrera de Ingeniería Empresarial en el contexto geográfico próximo, validando en este indicador el estudio de pertinencia y prospectiva de la oferta académica.

\section{Tabla 5}

\section{Pertinencia social y territorial de los emprendimientos, periodo 2012-2018. Carrera de Ingeniería Empresarial, a partir de la implementación.}

\begin{tabular}{ccc}
\hline \multicolumn{3}{c}{ IMPLEMENTACION } \\
Rango & Frecuencia & Porcentaje \\
\hline $\mathrm{Si}$ & 2 & $3 \%$ \\
No & 59 & $84 \%$ \\
No contesta & 9 & $13 \%$ \\
\hline Total & 70 & $100 \%$ \\
\hline
\end{tabular}

Fuente: Elaboración propia a partir del trabajo de campo realizado

La tabla 5 muestra que tan solo el $3 \%$ de los emprendimientos formulados mediante los trabajos de titulación son implementados, lo que afecta a la pertinencia social de la línea y ámbitos de investigación de la carrera de Ingeniería
Empresarial. El hallazgo contradice a los indicadores de pertinencia territorial y viabilidad económica y financiera que son favorables. Posiblemente existan factores asociados a la no implementación de los emprendimientos que tengan características formativas, culturales, emocionales y de barrera de acceso al financiamiento. Por tanto, se abre una nueva puerta de estudio correlacional entre estos posibles factores y su incidencia en la implementación de emprendimientos productivos interculturales con pertinencia social y territorial.

Tabla 6

Interculturalidad de los emprendimientos, periodo 2012-2018. Carrera de Ingeniería Empresarial, a partir de su enfoque

\begin{tabular}{ccc}
\hline \multicolumn{3}{c}{ INTERCULTURALIDAD } \\
Rango & Frecuencia & Porcentaje \\
\hline $\mathrm{Si}$ & 48 & $69 \%$ \\
$\mathrm{No}$ & 22 & $31 \%$ \\
\hline Total & 70 & $100 \%$ \\
\hline
\end{tabular}

Fuente: Elaboración propia a partir del trabajo de campo realizado

La tabla 6 evidencia desde el estudio de campo que los emprendimientos presentados como trabajos de titulación en un $31 \%$ aún no responden a un proceso de interculturalidad venido desde el diálogo de saberes. El hallazgo genera discusión desde la función sustantiva de 
vinculación con la sociedad y la estrecha relación con la investigación formativa y la pertinencia académica. Los proyectos de vinculación deben asumirse como vehículos de convergencia entre el conocimiento científico y el conocimiento cotidiano para a partir de esas realidades, desarrollar propuestas innovadoras que generen impacto y transformación social.

\begin{tabular}{ccc} 
Tabla 7 \\
Innovación de los \\
emprendimientos. Periodo \\
2012-2018. Carrera de \\
Ingeniería Empresarial. \\
\hline \multicolumn{3}{c}{ INOVACION } \\
Rango & Frecuencia & Porcentaje \\
\hline $\mathrm{Si}$ & 24 & $34 \%$ \\
No & 46 & $66 \%$ \\
\hline Total & 70 & $100 \%$ \\
\hline
\end{tabular}

Fuente: Elaboración propia a partir del trabajo de campo realizado

Apenas el $34 \%$ de los emprendimientos que provienen de los trabajos de titulación de la carrera de Ingeniería Empresarial, responden a innovación de proyecto e innovación de la gestión empresarial, dato relevante que se muestra en la tabla 7 . El dato prende una alarma que podría llevar a reformular el currículo en los niveles meso y micro, tomando en cuenta que el emprendimiento y la innovación caminan juntas con el desafío de generar cultura emprendedora e innovadora como eje transversal del cursado a nivel de grado.

De esta forma quedan presentados los hallazgos o resultados más relevantes de la presente investigación cuantitativa.

A partir de los hallazgos expuestos, estudios que se relacionan con la propuesta de investigación se encuentra en la experiencia del programa de fomento a la cultura emprendedora en el contexto de la educación superior en Gipuzkoa- País Vasco. Consiste en un modelo universitario relacional donde se distinguen categorías de cultura emprendedora como ideas $y$ perfiles de negocios, pre inversión y competencias transversales para creación de proyectos, lo que dialoga con la pertinencia social y territorial de los proyectos de emprendimiento innovadores que a su vez provienen del diálogo de saberes.

Se ha rastreado un texto sobre estudios aplicados en el ámbito del emprendimiento y la innovación con pertinencia social y territorial en el contexto canadiense sobre Iniciativa local y desarrollo: respuesta social a la globalización neoliberal. Se concluye que el territorio tiene una función de referente instituyente del tejido social vinculado a la pertinencia territorial de los involucrados, en un espacio temporal que supone la explotación racional de los recursos para el fomento de la producción con criterio de sostenibilidad y sustentabilidad planetaria, que sustenta a las ideas creativas dispuestas a correr riesgos.

Una interesante experiencia desde una mirada a las unidades económicas de los estudiantes y egresados de la Universidad EAN en el contexto colombiano aporta Parra y Argote (2015) al caracterizar el emprendimiento anclado a la malla curricular. El estudio se aplicó a 346 unidades económicas mediante un censo que se lo hizo de forma presencial y por el internet. Los resultados demuestran que los estudiantes y egresados tienen unidades económicas de su propiedad o administran empresas de sus padres. El tamaño de las empresas está condicionadas a que 
operan con menos de 10 empleados en su mayor porcentaje y se especializan en el sector servicios principalmente, lo equivale a los ejercicios de creatividad para transformar procesos a través del emprendimiento innovador.

De modo que si es posible fomentar la cultura emprendedora en las Universidades, no solamente en las carreras o facultades de Administración de Empresas, sino en forma general, en la medida que el emprendimiento y la innovación es transversal a toda la oferta académica universitaria, en diálogo con la generación de un impacto positivo y original de nuevos productos, bienes y servicios, con valor agregado para el consumidor cliente, en un contexto de transferencia del conocimiento intergeneracional con dignidad.

\section{Reflexiones finales}

El emprendimiento y la innovación desde el campo teórico, es amplio y refleja distintas posiciones y abordajes en contextos específicos de estudio. La breve revisión sistemática propone una línea de tiempo que parte de autores reconocidos por la comunidad epistémica como los iniciadores de la discusión científica como Schumpeter, pasando por autores seminales y contemporáneos.

Así mismo, el abordaje teórico de la pertinencia social y territorial toma auge en la producción científica aunque para el tema interculturalidad y diálogo de saberes el nivel aún es exploratorio, sin restar relevancia a lo dicho por autores como Jesús Martín Barbero quienes se sitúan en el punto de inflexión entre el conocimiento científico y el conocimiento cotidiano.

El enfoque metodológico aporta núcleos duros al estar bajo el paradigma positivista que persigue el dato concreto de la realidad abstracta. Se mueve en función del método científico y promueve técnicas e instrumentos validados para el análisis y discusión de los datos tomados de la bitácora de trabajos de titulación de la carrera de Ingeniería Empresarial.

El emprendimiento y la innovación en el ámbito universitario constituyen un eje de acción sistémica. El ordenamiento legal de la educación superior en el Ecuador establece como parte de las funciones sustantivas el principio de pertinencia social, territorial y ambiental. En este sentido, la carrera de Ingeniería Empresarial de la Universidad Católica de Cuenca Sede Azogues, desarrolló decenas de proyectos de emprendimiento a nivel de perfil o pre inversión como requisito de grado, previa su titulación, en el periodo 20122018. En correspondencia, la innovación se sitúa en un momento de arranque y posicionamiento, con un indicador que implica mejoramiento tanto a nivel producto y a la gestión empresarial.

Los perfiles de emprendimientos se hallan en correspondencia con la vocación económica y productiva del área de influencia de la Carrera de Ingeniería Empresarial y se originan en las salidas productivas de los planes de desarrollo y ordenamiento territorial en los distintos niveles, lo que consolida su pertinencia territorial. Sin embargo, la implementación del diseño de los modelos de negocios, es marginal. Lo que se ve reflejado en el indicador de pertinencia social. El resultado así presentado por las limitaciones tempo espaciales del estudio no aclara los factores que inciden en la pertinencia social de los emprendimientos de la carrera de Ingeniería Empresarial, lo que 
genera nuevas líneas de averiguación más profundas con enfoque correlacional y explicativo.

En correspondencia con la pertinencia territorial, la interculturalidad es una categoría de alta incidencia en los emprendimientos estudiados, lo que conlleva, a su fortalecimiento como una dimensión social inclusiva con indicadores de mercado, técnicos, económicos y financieros favorables.

En resumen, los precedentes abalizan la necesidad de enriquecer y potenciar la línea y los ámbitos de investigación en la medida que garanticen la implementación de los emprendimientos innovadores con impacto social en el área de influencia y vocación económica, productiva, cultural y ambiental de la carrera de Ingeniería Empresarial de la Universidad Católica de Cuenca Sede Azogues.

\section{Referencias bibliográficas}

Castillo, Alicia (1999), Estado del arte en la enseñanza del emprendimiento. Emprendedores como creadores de riqueza y desarrollo regional. First Public Inc Chile S.A. en 1999 para INTEC-CHILE

Fernández, Marco (1997), Gestión tecnológica para un salto en el desarroIlo industrial, España. CDTI-CSIC.

Formichella, María (2004), El concepto de emprendimiento y su relación con la educación, el empleo y el desarrollo local. Revista Chacra Experimental Integrada Barrow. Argentina.

Gee, Sherman (1981), Technology transfer, innovation \& international competitiveness. Wiley and Sons, New York.

Grigsby, Anderson (2010), Qué pasará en las elecciones con una economía tan incierta y tan austera. Revista Envío, Non238, pp 1-5.

Jiménez, Angélica; Hernández Palma, Hugo G.; Pitre Redondo, Remedios (2018), Emprendimiento social y su repercusión en el desarrollo económico desde los negocios inclusivos.

Revista Logos Ciencias y Tecnología. No. 10 Vol. 2, pp 1-15.

Jiménez-Silva, Walter; Larrea-Altamirano Janeth; Navarrete-Fonseca Mario; Castro-Ayala Edisson (2019), Emprendimientos innovadores a partir de competencias cognitivas en estudiantes universitarios. Revista Venezolana de Gerencia. No. 24, Vol. 85, pp. 1-15. Universidad del Zulia, Maracaibo - Venezuela. Disponible en: http://www.produccioncientificaluz.org/

Presidencia de la Republica (2018). Ley 0 . Ley Orgánica de Educación Superior. Registro Oficial Suplemento 298. Ecuador.

Barbero, Martín Jesús (2008), Un mapa para la interculturalidad. Revista Latinoamericana de Comunicación Chasqui. No. 102, pp 14-27.

Naidorf, Judith; Giordana, Patricia; Horn, Mauricio (2007), La pertinencia social de la Universidad como categoría equívoca. Revista Nómadas, No. 27, pp 22-33. Universidad Central Bogotá, Colombia

Nelson, Richard y Winter, Sidney (1982), An evolutionary theory of economic change. Harvard University Press, Cambridge.

OECD/Eurostat (2018), Manual de Oslo: Guidelines for Collecting, Reporting and Using Data on Innovation, 4th Edition, The Measurement of Scientific, Technological and Innovation Activities, Paris/Eurostat, Luxembourg. 
Parra, León y Linneth, Milenka (2015), Una mirada a las empresas de los estudiantes y egresados: el caso de la Universidad EAN. Revista Estudios Gerenciales. No.31, pp. 122134

Pavón, Julián e Hidalgo, Antonio (1997), Gestión e innovación: un enfoque estratégico, España. Ediciones Pirámide.

Pavón, Julián y Goodman, Richard (1981), La planificación del desarrollo tecnológico. CDTI-CSIC, España, Proyecto MODELTEC.

Rodríguez, Alfonso (2009), Nuevas perspectivas para entender el emprendimiento empresarial. Revista Pensamiento y Gestión, No. 26, pp 1-94.

Schumpeter, Joseph (1934), The Theory of Economic Development: An Inquiry into Profits, Capital, Credit, Interest and the Business Cycle, Harvard University Press, Cambridge, MA.

Schumpeter, Joseph (1984), The meaning of rationality in the social sciences. Zeitschrift für die gesamte Staatswissenschaft/Journal of Institutional and Theoretical Economics No. 4, pp 577-593.

Secretaria Nacional de Planificación y
Desarrollo (2017), Plan Nacional de Desarrollo, Toda una Vida. Recuperado en línea el 15/07/2019 en: https:// www.planificacion.gob.ec/wp-content/uploads/downloads/2017/10/ PNBV-26-OCT-FINAL_OK.compressed1.pdf

Universidad Católica de Cuenca (2016), Líneas, ámbitos de investigación desde los dominios académicos institucionales. Recuperado en línea el 15/07/2019 en: https://documentacion.ucacue.edu.ec/files/original/ c2548b2e061670d41a1801c251ddeb09.PDF

Vicente, Miguel y Meléan, Rosana (2018), Empresas Pyme o Empresarios Pyme. Revista Venezolana de Gerencia. 23(82), pp 1-6. Universidad del Zulia, Maracaibo - Venezuela.

Zafra Tristancho, Sandra Liliana; Martínez Lozano, José Joaquín; Vergel Ortega, Mawency (2014), Indicadores para evaluar la pertinencia social en la oferta académica de programas. Revista Logos Ciencia \& Tecnología. No. 6, Vol.1, pp 1-15. Policía Nacional de Colombia Bogotá, Colombia

- Esta obra está bajo una licencia de Creative Commons Reconocimiento-NoComercialCompartirlgual 3.0 Unported.

http://creativecommons.org/licenses/by-nc-sa/3.0/deed.es_ES 


\title{
Calidad de servicio en el proceso de recolección domiciliario de residuos sólidos
}

\author{
Sáez Molero, Alejandrina Luz ${ }^{1}$ \\ Urdaneta Graterol, Joheni Alejandra²
}

\section{Resumen}

La presente investigación tiene como objetivo diseñar un instrumento válido que permita medir la calidad de servicio del proceso de recolección domiciliario de residuos sólidos del Municipio Maracaibo. La investigación es de tipo descriptiva ya que se establecen las dimensiones que miden la calidad percibida por los usuarios del servicio. En el estudio se trabaja con una prueba piloto en la que se toma una muestra no probabilística circunstancial de 50 usuarios del servicio. Se diseña un instrumento cerrado, tipo escala, basado en los reactivos del Service Performance (SERVPERF), adicionando la dimensión participación ciudadana; dicho instrumento es sometido a validación de contenido y cuantitativa, estableciendo su consistencia interna, validez de constructo, criterio, estabilidad y rendimiento. Cómo resultado se obtiene un instrumento con una confiabilidad de 0,845; que presenta cuatro dimensiones: comunicación, capacidad de respuesta, frecuencia y horarios de recolección y elementos tangibles, que permiten medir la calidad del servicio. Presenta además validez de criterio y un rendimiento del 0,834 . Se concluye que el instrumento diseñado es confiable, valido y está adaptado para la población objeto de estudio.

Palabras clave: calidad de servicio; proceso de recolección domiciliario; residuos sólidos; análisis factorial; SERVPERF.

Recibido: 31-05-18 Aceptado: 18-02-19

Ingeniera Industrial (LUZ). Magister en Gerencia de Empresas: Mención Mercadeo (LUZ). Profesor Titular de la Facultad de Ingeniería, Universidad del Zulia. Investigador responsable del proyecto: CALIDAD DEL ASEO URBANO EN LA CIUDAD DE MARACAIBO. Aprobado y financiado por el CONDES. Teléfono: 02614128786. E-mail: asaez@fing.luz.edu.ve. Autor para la correspondencia.

2 Ingeniera Industrial (LUZ). Doctora en Ciencias Sociales (LUZ). Profesor Titular de la Facultad de Ingeniería, Universidad del Zulia. Investigadora responsable del programa: Calidad de los servicios públicos en la región zuliana. Aprobado y financiado por el CONDES. E-mail: jhurdaneta@fing.luz.edu.ve 


\title{
Service quality in the process of domiciliary collection of solid waste
}

\begin{abstract}
The objective of this research is to design a valid instrument that allows to measure the service quality of the municipal solid waste collection process in the Maracaibo Municipality. This research is of the descriptive type, as the dimensions that measure the quality perceived by the users of the service are established. In the study, a pilot test is used, in which a non-probabilistic circumstantial sample of 50 service users is taken, A closed, scale-type instrument is designed, based on the SERVPERF reagents, also including the dimension and citizen participation; such instrument is subject to content and quantitative validation, establishing its internal consistency, construct validity, criteria, stability and performance. The instrument obtained as a result has a reliability of 0.845 , and shows four dimensions: communication, response capacity, frequency and collection schedules, and tangible elements that allow to measure the quality of the service. It also presents criterion validity and a performance of 0.834 . It is concluded, then, that the designed instrument is reliable, valid and adapted to the population under study.
\end{abstract}

Keywords: quality of service; home collection process; solid waste; factor analysis; SERVPERF.

\section{Introducción}

La calidad es un factor de suma importancia para todas las organizaciones, en especial para las empresas que prestan servicios públicos, ya que en la medida que los ciudadanos reciban servicios eficientes, mejora su calidad de vida, por lo tanto, saber cuál es la calidad percibida por los usuarios es clave ya que permite mejorar la prestación de estos servicios.

Son diversos los modelos que se han diseñado con la finalidad de medir la calidad de los servicios, uno de ellos es el modelo de las deficiencias conceptualizado por S. Parasuraman, Valerie Zeithaml y Berry, entre 1985 y 1994; otro, es el modelo nórdico de Grönroos presentado en 1990, el cual señala que la calidad percibida por los clientes es el resultado de la integración de la calidad técnica (qué se da) y la calidad funcional (cómo se da) con su relación con la imagen corporativa; en este modelo, la imagen es un elemento clave para medir la calidad percibida (Duque Oliva, 2005)

De estos modelos, el más conocido y aplicado es el modelo de las deficiencias, el cual se concreta en un instrumento denominado Service Quality (SERVQUAL) que constituye una herramienta para medir la calidad de un servicio a través de la diferencia entre las percepciones que tienen los clientes respecto al servicio y sus expectativas reales. Es un instrumento de escala múltiple que presenta un alto nivel de fiabilidad y validez. El modelo incluye dos dimensiones de las expectativas: las expectativas deseadas y las expectativas adecuadas. 
Otro instrumento utilizado para medir la calidad de un servicio es el SERVPERF, este consiste en una escala centrada en la medición de la calidad percibida de los servicios a través de la satisfacción del cliente. El instrumento fue desarrollado por Cronin y Taylor, quienes probaron un modelo alternativo sustentado en el desempeño, el cual se basa en 22 de los ítems propuestos en el SERVQUAL y que sugieren mesurar la calidad solo con el test de percepciones que propone el mismo. Según las pruebas practicadas por Cronin y Taylor, la escala SERVPERF parece conformar más de cerca las implicaciones de la literatura sobre actitudes y satisfacción (Duque Oliva, 2005).

En referencia a los servicios públicos Bigné, Moliner, Vallet, y Sánchéz (1999) indican que fue a finales de la década de los 80 cuando comienzan a presentarse investigaciones orientadas a estudiar la calidad de los servicios públicos. En muchas investigaciones se han utilizado el SERVQUAL y el SERVPERF para valorar la calidad de estos; por ejemplo, los citados autores, comparan SERVQUAL y SERVPERF en 888 individuos repartidos en tres servicios públicos diferentes: hospitalarios, universitarios y ferroviarios, determinando la validez y confiabilidad de ambos instrumentos y verificando que la escala SERVQUAL es más confiable que la escala SERVPERF, así mismo ratifican las cinco dimensiones propuestas por Parasuraman, Valerie Zeithaml y Berry.

En otras investigaciones se han utilizado las escalas mencionadas obteniéndose resultados diferentes, Montaña, et al, (2002) evaluaron la calidad de los servicios prestados por las empresas de servicios públicos domiciliarios de agua, energía, teléfono y gas utilizando la escala SERVPERF ratificando que es confiable y definiendo dimensiones diferentes para cada tipo de servicio con el análisis factorial, es decir, no se confirman las dimensiones originales del modelo.

Por su parte Maldonado etal, (2013) presentan una perspectiva interesante cuando afirman que cada servicio se presta en contextos muy específicos lo que hace necesario adaptar los modelos para que sean capaces de efectuar una medición real del servicio al considerar aspectos culturales que pueden interferir significativamente en la percepción del consumidor.

Bajo esta premisa, la de diseñar un instrumento adaptado a la cultura Marabina, se toma como base la escala SERVPERF y se le adiciona una dimensión denominada: Participación Ciudadana, la cual se considera importante por tratarse de un servicio público. Se utiliza el análisis factorial exploratorio para la determinación de los factores que realmente miden la calidad de servicio del proceso citado a través de la percepción de los usuarios marabinos.

La investigación realizada corresponde a la segunda fase de un proyecto por lo que la misma es de tipo descriptiva ya que se logra establecer el conjunto de factores subyacentes que soportan la variable objeto de estudio en el servicio observado; la investigación de tipo descriptiva según Monje Álvarez (2011:95) "es aquella en la cual se procede con base en la información obtenida, a ordenar los rasgos, atributos o características de la realidad observada de acuerdo con el problema investigativo planteado". El diseño de la investigación es no experimental transeccional en virtud de que las varibles independientes no fueron manipuladas y las mediciones se tomaron en un solo momento en el 
tiempo (Hernández et al,: 2006).

Una vez establecido el objetivo de la investigación y la variable objeto de estudio, se procedió a diseñar la escala tomando los reactivos de la escala SERVPERF y adaptando la redacción de los mismos al servicio cuya calidad desea medirse. El instrumento quedo conformado por 27 reactivos, medidos con la escala Likert, que explican seis dimensiones de la calidad de servicio: las cinco definidas por S. Parasuraman, Valerie Zeithaml y Berry y una adicionada por los investigadores en virtud de que el servicio estudiado es un servicio público.

Con la finalidad de validar el contenido y estructura del instrumento, se sometió a juicio de tres expertos en el área de calidad de servicio, quienes evaluaron el instrumento de manera independiente. Acada experto se le dieron dos criterios para evaluar el instrumento, la pertinencia de los reactivos con los objetivos de investigación y contenido teórico, y la redacción de los mismos.

Para continuar con la validación del instrumento diseñado, se aplicó el mismo a un conjunto de individuos con características similares a los sujetos que conforman la población objeto de estudio. En total debía encuestarse un mínimo de 50 personas o sujetos, los cuales contaban con las siguientes características: ser mayores de edad, jefes del grupo familiar que vive en el inmueble visitado y recibir el servicio de recolección domiciliario de residuos sólidos. El mínimo de sujetos a encuestar se establece en función de que el instrumento cuenta con 27 reactivos o ítems, y 54 es el doble de estos, garantizando de esta manera que la cantidad de datos cumple con las consideraciones especiales planteadas por García et al, (2013) quienes señalan que en las pruebas piloto se recomienda incluir entre 30 a 50 participantes los cuales deben poseer los atributos que se desean medir en la población objetivo.

Una vez revisado el instrumento por los expertos y aplicado a los sujetos de la prueba piloto, se detectaron algunos problemas de redacción e interpretación de los items, asi como reactivos que no discriminaban, y adicionalmente la confiabilidad (Alfa de Cronbach) fue apenas de 0,689 valor que se considera no adecuado.

Estos resultados llevaron a redefinir el instrumento, realizándose una nueva revisión teórica que complementada con las sugerencias de los expertos llevo a estructurar una escala con el mismo número de ítems por dimensión. Se modificaron las redacciones de los ítems no comprendidos y de aquellos que no discriminaban y se adicionaron tres reactivos sugeridos por los expertos.

Para la nueva versión del instrumento se determina el tamaño de muestra para la prueba piloto en función de los recursos (encuestadores y tiempo) disponibles para la recolección de la data y con base en lo que plantean García et al, (2013); se seleccionan entonces, por muestreo no probabilístico, una primera muestra de aproximadamente 50 representantes de hogares que reciben el servicio en ocho parroquias diferentes del municipio Maracaibo, a saber: Cristo de Aranza, Juana de Ávila, Francisco Eugenio Bustamante, Chiquinquirá, Bolívar, Caracciolo Parra Pérez, Cacique Mara y Olegario Villalobos, para la aplicación del Análisis Factorial (AF). Una segunda muestra de 38 usuarios para la verificación de la validez de criterio y una tercera muestra de 13 sujetos para verificar la reproducibilidad del instrumento.

Los datos recolectados con esta nueva versión, permiten establecer la 
confiabilidad y validez del instrumento, lo cual se presenta en las secciones siguientes, comenzando con la definición de las dimensiones que fueron establecidas en el constructo teórico para medir la calidad de servicio del proceso de recolección domiciliario de residuos sólidos prestado en el municipio Maracaibo.

\section{Diseño del instrumento/ escala de medición}

Referente al termino escala, Lévy y Varela (2003:45) la definen como un determinado tipo de instrumento de medida utilizado con mucha frecuencia en el ámbito de las ciencias sociales, concretamente indican que son "instrumentos que pretenden cuantificar características en las que no hay respuestas correctas 0 incorrectas, como ocurre en la medición de atributos relacionados con las actitudes, opiniones, intereses, disposiciones o personalidad."

En la presente investigación, el término escala está referido al instrumento con el cuál será posible recabar la información concerniente a la percepción, que tiene el usuario del servicio de recolección domiciliario de residuos sólidos, para utilizarla con el fin de medir la calidad del servicio.

Para diseñar la escala es importante resaltar que se parte de la hipótesis de que la percepción que tienen los usuarios del servicio de recolección domiciliario de residuos sólidos permite medir la calidad del mismo.

En virtud de esta hipótesis se hace necesario contextualizar las dimensiones que permiten medir la calidad para el servicio estudiado.

\subsection{Calidad de servicio del proceso de recolección domiciliario. Dimensiones}

En función de que el propósito de la investigación es diseñar un instrumento válido para medir la calidad subjetiva del servicio de recolección domiciliario de residuos sólidos a través de las percepciones de los usuarios, se toma como referencia para el diseño de la escala el modelo basado en el desempeño, específicamente el instrumento diseñado por Cronin y Taylor, SERVPERF, el cual está fundamentado en el modelo propuesto por Parasuraman, Zeithaml y Berry, entre 1985 y 1994.

EI SERVPERF está estructurado por 22 ítems que se centran en medir la percepción que tiene el usuario o cliente, referente al servicio que recibe; estos ítems se agrupan en las cinco dimensiones de calidad propuestas por Parasuraman y sus colaboradores.

Para el instrumento de valoración de la calidad de servicio del proceso de recolección domiciliario de residuos sólidos se consideran seis dimensiones: las cinco primeras basadas en el SERVPEF y adaptadas a las necesidades particulares del servicio, diseñadas básicamente para cuantificar la calidad del servicio acreditable a la institución encargada de la prestación del mismo (factores internos), y una sexta dimensión que busca medir la calidad subjetiva del servicio imputable a factores externos a la institución, específicamente el usuario.

Las dimensiones establecidas para el proceso de recolección domiciliario se definen a continuación:

Elementos tangibles: para los creadores del modelo esta dimensión de 
la calidad está referida a la apariencia de las instalaciones físicas, equipos, personal y materiales de comunicación asociada al servicio. En la prestación del servicio recolección domiciliario de residuos sólidos los aspectos tangibles que captan los usuarios son:

- Condiciones de los camiones, específicamente si estos presentan un estado o aspecto aceptable, es decir que no estén en malas condiciones ni sea palpable un mal funcionamiento de los mismos; otro factor importante es la identificación del vehículo con el logo de la empresa o institución que presta el servicio.

- Apariencia del personal recolector de basura, lo mínimo requerido en este aspecto es el uso de un uniforme que identifique al personal como miembro de la institución, y que éste se encuentre en condiciones aceptables.

- Aspecto del material publicado por el instituto que presta el servicio exponiendo a los usuarios del servicio temas relacionados con el mismo, tales como: horarios, frecuencias, normas para disposición de residuos, etc.

Confiabilidad: según

Parasuraman et al, (1992:29) "es la habilidad para prestar el servicio prometido de forma cuidadosa y fiable, es decir, que la empresa entregue el servicio que promete, y que preste bien el servicio desde el comienzo". Respecto a esta dimensión de la calidad para el servicio de recolección domiciliario de residuos sólidos, se consideran los siguientes aspectos:

- La credibilidad del instituto que presta el servicio al cumplir a los usuarios el servicio prometido.

- Cumplimiento de responsabilidades por parte del personal recolector al realizar su trabajo garantizando no esparcir residuos en las calles.

- Cumplimiento de responsabilidades por parte del instituto al efectuar la recolección con la frecuencia prometida, en los días fijados en la semana para cumplir con dicha frecuencia y los horarios establecidos para tal fin.

- Información oportuna por parte del instituto con la finalidad de mantener enterado al usuario de la frecuencia de recolección en su sector, los días a la semana y horario en que será cumplida esa frecuencia.

Capacidad de respuesta: definido por Zeithaml et al, (1993:29) como "la disposición y voluntad de los empleados para ayudar a los clientes y ofrecerles un servicio rápido". Para el servicio de recolección domiciliario de residuos sólidos resulta clave considerar en esta dimensión de la calidad aspectos tales como:

- Atención rápida y oportuna de las necesidades y demandas planteadas por los usuarios.

- Interés mostrado por el instituto que presta el servicio para atender y solucionar los problemas de los usuarios y comunidades, el cual se evidencia en la disposición y disponibilidad de recursos como: canales, sistemas o mecanismos para recibir reclamos, empleados y trabajadores con actitud proactiva para atender las quejas, reclamos y solicitudes de las comunidades y usuarios.

Seguridad: conocimientos y atención mostrados por el personal de contacto y sus habilidades para inspirar credibilidad y confianza al cliente. El servicio de recolección domiciliario de residuos sólidos tiene la característica 
particular que la relación directa entre el prestador del servicio y el usuario en muchas ocasiones no se presenta. Por esta razón la dimensión seguridad se define bajo las siguientes premisas:

- Confianza que genera la institución evitando riesgos para sus clientes, haciendo que estos se sientan tranquilos y no perciban peligros en su relación con el proveedor de servicio, tanto en la ejecución de la recolección, como en las transacciones que realizan entre ellos.

- Credibilidad en la institución contemplada en el conocimiento sobre el servicio (horarios, frecuencia, como proceder a la hora de un reclamo, entre otras) que tiene el personal recolector y su comportamiento con el usuario referido específicamente al respeto en el trato y al cumplimiento de los procedimientos.

Empatía: es la atención individualizada que el proveedor del servicio ofrece a los clientes. Resulta claro que por el tipo de servicio es difícil establecer una atención individualizada por usuario, por lo tanto, se consideran para esta dimensión de la calidad aspectos como:

- Comprensión de las necesidades de los usuarios respecto al servicio: referido al establecimiento de horarios y frecuencias en función de la generación de desechos, comunicación de las modificaciones de la programación en la prestación del servicio, entre otras.

- Interés mostrado por el instituto para procesar y/o canalizar reclamos, quejas y opiniones de los usuarios.

La última dimensión incluida para cuantificar la calidad de servicio del proceso de recolección domiciliario de residuos sólidos en el municipio Maracaibo busca medir la calidad a través de la percepción del usuario referente a su participación en el proceso.

Participación Ciudadana: se incluye esta dimensión por considerar de vital importancia la colaboración del usuario en la mejora continua del proceso de recolección, al incidir de forma directa con su intervención en la calidad del mismo.

Ceballos (2009:45) expresa que "etimológicamente la palabra participación proviene del latín "partem capere" que se traduce en "tomar una parte" o " tomar parte"; en sentido técnico la participación ciudadana es comprendida como un proceso social, continuo y dinámico, por medio del cual los miembros de una comunidad a través de mecanismos establecidos y organizaciones legítimas en las cuales se encuentren representadas todos los miembros de la comunidad deciden, aportan y participan en la realización del bien común."

Para el Consejo Legislativo del Estado Zulia (2004) la participación ciudadana "se entiende como la intervención de los ciudadanos, individualmente o por intermedio de sus asociaciones u organizaciones sociales, en los procesos de formulación, ejecución, seguimiento, control y evaluación de las políticas públicas"

La participación en los asuntos comunitarios, se refiere a la intervención de los vecinos, individualmente $o$ a través de sus organizaciones sociales, con el propósito de atender los problemas comunes y satisfacer sus necesidades y demandas de servicios, coadyuvando con el Gobierno del Estado Zulia a adoptar acciones tendentes al mejoramiento de su calidad de vida."

Una definición similar se presenta 
en el proyecto de Ley Orgánica de Participación Ciudadana y Poder Popular (2001) "se entiende por participación ciudadana, protagónica y corresponsable, la disposición consciente de las personas a involucrarse de manera individual o colectiva en la formulación, proyección, ejecución, control social y evaluación de las políticas públicas, que permitan la consolidación constitucional de un Estado democrático y social de derecho y de justicia."

En función de los objetivos se delimita el concepto de participación ciudadana a aquellos aspectos que puedan medirse con la percepción del usuario del servicio y que adicionalmente estén conceptualizados para el mismo, es decir, que actualmente estén establecidos ya sea por leyes, ordenanzas o normativas declaradas por alguna institución gubernamental u oficial.

Para la presente investigación, se define la participación ciudadana como la disposición de los usuarios del proceso de recolección domiciliario de residuos sólidos a intervenir en la ejecución y control del mismo, cumpliendo con los deberes que le impone la ley, exigiendo el cumplimiento de sus derechos y colaborando con el instituto recolector de desechos.

Se consideran para esta dimensión los siguientes aspectos:

- Conocimiento de sus deberes y derechos como usuario del servicio, aspecto que les permite evaluar y controlar la gestión del instituto que presta el servicio.

- Conocimiento sobre las regulaciones del servicio, esto promueve su colaboración con el instituto recolector de desechos sólidos al indicarle cuál es su rol como usuario del servicio.

- El conocimiento sobre el manejo de residuos sólidos constituye un factor importante en la calidad del servicio, ya que facilita la ejecución del servicio e incide en la conservación del medio ambiente.

Para concretar la estructura y forma del instrumento se procede a diseñar los ítems que llevan a medir la calidad del servicio de aseo urbano.

\subsection{Elaboración de los ítems.}

Como ya se expuso, se adapta (para medir la calidad de servicio del proceso de recolección del aseo urbano) la escala diseñada para el SERVPERF, redactando enunciados orientados a cuantificar cada dimensión de calidad y adicionando indicadores que permitan medir la participación ciudadana, como factor que incide en la calidad del servicio.

El instrumento que se propone queda conformado por 30 enunciados, 15 de los cuales están redactados de forma positiva y 15 se redactan de forma negativa, lo cual se hace "para evitar la tendencia reactiva a responder en una sola dirección, por ejemplo, sin detenerse a leer responder "totalmente de acuerdo" a todos los ítems" (Blanco, 2000:63). Para cada dimensión se redactan 5 ítems o reactivos, los cuales son codificados con una escala tipo Likert. El instrumento diseñado se denota como CASERAU (calidad de servicio del proceso de recolección del aseo urbano), y será referenciado con ese nombre de aquí en adelante.

\subsection{Fiabilidad y validez del instrumento}

Un instrumento debe ser válido 
y confiable para poder ser utilizado en la recolección de datos. Por lo tanto, todo instrumento diseñado a partir de un constructo teórico o adaptado de un instrumento extranjero, debe aplicarse a la población objeto de estudio en una prueba piloto para verificar su confiabilidad y validez.

La validez total se gana con una mayor validez de contenido, validez de constructo y validez de criterio en el instrumento de medición (Paniagua, 2015:2).

Una vez realizada la revisión teórica y culminado el diseño del instrumento en la presente investigación y previa validación de contenido del mismo, se estudia su confiabilidad y validez, para lo cual se evalúan: confiabilidad o consistencia interna, validez de constructo, criterio, estabilidad y rendimiento de la escala.

\section{- Confiabilidad}

La seguridad o fiabilidad de una escala la define Lagunes (2017) como la propiedad de proporcionar resultados similares en sus sucesivas aplicaciones. Por su parte Levy y Varela (2003) expresan que la fiabilidad de una escala se refiere a la precisión de las puntuaciones que esta ofrece. Si una escala es fiable las puntuaciones obtenidas para un determinado grupo de personas constituirán valores que serán replicables siempre que se mantengan inalteradas las condiciones de administración de la escala.

Ambas definiciones enuncian que una escala o instrumento es confiable si al aplicarse, en condiciones idénticas, las puntuaciones o mediciones obtenidas son similares o tienden a una misma apreciación del criterio o atributo medido.

Una de las estrategias utilizadas para medir la confiabilidad de una escala radica en medir la consistencia interna entre los ítems, la cual hace referencia al grado de relación existente entre los mismos (Arribas Martín, 2004). Entre los métodos más utilizados para medir la consistencia interna de una escala se encuentra el del Coeficiente de Cronbach $(\alpha)$ el cual según Levy y Varela (2003) permite resumir de una manera sencilla la correlación entre los ítems. Este coeficiente asume valores entre 0 y 1 , considerándose que existe una buena consistencia cuando tiene valores mayores a 0,70 (Arribas Martín, 2004).

La confiabilidad obtenida en la prueba piloto para el instrumento conformado por 30 ítems fue de 0,756, lo que se considera alto (Arribas Martín, 2004; Levy y Varela, 2003).

Se realiza un análisis de la correlación entre los reactivos del instrumentos y la variable estudiada (Calidad de servicio), obteniéndose que un total de 9 ítems presentan correlaciones muy bajas con ella (tabla 1). Los resultados de las pruebas de correlación muestran coeficientes de Pearson ( $\rho$ ) muy bajos y valores $P$ mayores a 0,05 lo que lleva a concluir que no existe correlación significativa entre cada uno de los ítems y la calidad de servicio. Aunado a esto, para cada uno de los ítems, se observa que al ir eliminandolos el Alpha de Cronbach va mejorando. 


\section{Tabla 1}

Resultados de las pruebas de correlación entre los ítems y la variable Calidad de Servicio y su impacto en el Alpha de Cronbach una vez eliminados

\begin{tabular}{|c|c|c|c|}
\hline \multirow[b]{2}{*}{ ITEM } & \multicolumn{2}{|c|}{ PRUEBA CORRELACIÓN } & \multirow{2}{*}{$\begin{array}{c}\text { ALFA DE } \\
\text { CRONBACH }\end{array}$} \\
\hline & $\begin{array}{l}\text { COEFICIENTE } \\
\text { DE PEARSON }\end{array}$ & VALOR P & \\
\hline 1 & 0,212 & 0,14 & 0,777 \\
\hline 23 & 0,024 & 0,87 & 0,786 \\
\hline 20 & 0,052 & 0,722 & 0,795 \\
\hline 2 & 0,026 & 0,858 & 0,806 \\
\hline 30 & 0,037 & 0,801 & 0,812 \\
\hline 4 & 0,058 & 0,69 & 0,821 \\
\hline 28 & 0,038 & 0,796 & 0,838 \\
\hline 25 & 0,207 & 0,148 & 0,841 \\
\hline 19 & 0,227 & 0,112 & 0,845 \\
\hline
\end{tabular}

Fuente: elaboracion propia

Se decide eliminar los reactivos considerando lo siguiente: 1) la mayoría de los ítems requieren que el usuario entre en contacto directo con el personal recolector, situación que poco sucede, 2) se presenta una mejora en el indice de confiabilidad y 3) aumenta la facilidad de aplicación de la escala.

Se obtiene entonces una escala de 21 reactivos con una confiablidad de 0,845 , que permite obtener una puntuación mínima de 21 (muy baja calidad del servicio) y una puntuación máxima de 105 (muy alta calidad del servicio).

\subsubsection{Validez de constructo}

En esta fase de la elaboración del instrumento se busca establecer si los datos soportan o validan la teoría bajo la cual fue estructurado. Para el diseño de CASERAU, se elaboran los ítems basándose en las dimensiones que según la teoría propuesta por Zeithaml et al, (1993) miden la calidad de un servicio. Adicional a las cinco dimensiones presentadas por esta teoría se consideró adecuado, dadas las características particulares del servicio de aseo urbano, incluir una sexta dimensión denominada Participación Ciudadana, la cual busca 
medir la incidencia de la colaboración que deben prestar los usuarios del servicio de recolección para que este pueda efectuarse de manera apropiada.

La inclusión de una nueva dimensión a la teoría de calidad de servicio hace que sea necesario la realización de un Análisis Factorial Exploratorio (AF) para establecer cuales son los factores, que según la población objeto de estudio, inciden sobre la calidad de servicio del proceso de recolección domiciliario en el municipio Maracaibo.

Para la aplicación del AF se requiere el cumplimiento de cuatro supuestos básicos para las variables objeto de estudio: normalidad, varianza constante (homocedasticidad), independencia estadística y linealidad. En el caso concreto del análisis factorial la evaluación de estos supuestos puede reemplazarse por el análisis de las correlaciones entre las variables, considerando el hecho de que el no cumplimiento de dichos supuestos incide en una reducción de las correlaciones.

Hair et al, (2008) expresan que los supuestos básicos subyacentes del análisis factorial son más de tipo conceptual que estadístico e indican que el supuesto de normalidad sólo es necesario cuando se aplican pruebas estadísticas a la significación de los factores. Sugiere el autor que es recomendable cierto grado de multicolinealidad, dado que el objetivo de la técnica es identificar series de variables interrelacionadas.

Se inicia la evaluación de los supuestos revisando la matriz de correlación parciales entre las variables, estas miden la correlación entre dos variables considerando los efectos en ellas de otras variables. Según Hair et al, (2008: 88) "si los factores verdaderos existen en los datos, la correlación parcial será pequeña, porque se puede explicar la variable mediante los factores (valores teóricos con cargas para cada variable)". La presencia de correlaciones parciales altas, indica la inexistencia de factores subyacentes verdaderos $y$ en este caso la aplicación del análisis factorial resulta inadecuada.

Las correlaciones parciales entre las variables se logran con la ayuda del PASW.18, el cual arroja todas las correlaciones parciales en la Matriz de Correlaciones Anti-Imagen, en los resultados se observa un gran número de correlaciones parciales bajas, en total, un $88,57 \%$ de las mismas están por debajo de 0,3.

Seguidamente se utiliza el PASW.18 para aplicar el método de componentes principales con la finalidad de obtener los indicadores del grado de asociación entre ítems (los cuales se espera confirmen la existencia de intercorrelación entre los reactivos), los componentes que explican la variable estudiada y el porcentaje de varianza de la variable que estos expresan.

Los resultados obtenidos se muestran en la tabla 2, observándose un determinante de la matriz de correlaciones de $4,99{ }^{*} 10^{-6}$ el cual es bajo y cercano a cero, demostrando que existe intercorrelación entre los items; de igual manera la prueba de esfericidad de Bartlett soporta que existe correlación entre los reactivos ya que lleva al rechazo de la hipótesis de correlacón nula entre ítems dado que el Valor $\mathrm{P}=$ 0 . Es importante resaltar que el $\mathrm{KMO}$ resulta ser 0,572 valor considerado, según Carvajal et al, (2011), como no adecuado. A pesar de este último resultado, el cumplimiento de dos de los tres indicadores de asociación entre ítems, permite continuar con la aplicación del Análisis Factorial. 


\section{Tabla 2}

Determinante de la matriz de correlaciones, prueba de esfericidad de Bartlett y KMO

\begin{tabular}{|c|c|c|}
\hline \multicolumn{3}{|c|}{ Matriz de correlaciones (a) } \\
\hline \multicolumn{3}{|c|}{ a. Determinante $=4,99 \mathrm{E}-006$} \\
\hline \multicolumn{3}{|c|}{ KMO y prueba de Bartlett } \\
\hline Medida de adecua & uestral de Kaiser-Meyer-Olkin. & 0,572 \\
\hline Prueba de & Chi-cuadrado aproximado & 502,579 \\
\hline esfericidad de & Grados de libertad & 210,00 \\
\hline Bartlett & Valor $\mathrm{P}$ & 0,00 \\
\hline
\end{tabular}

La aplicación del método de componentes principales, con ayuda del PASW.18, arroja que son 4 las dimensiones subyacentes de la variable con una varianza explicada del $54,30 \%$, lo que se muestra en la tabla 3. Al realizar la revisión de las cargas factoriales, se hace evidente que para este resultado son muchos los items que cargan en la primera dimensión, ya que la misma explica por sí sola el $25,807 \%$ de la varianza de la variable.

Tabla 3

Porcentaje de la varianza de calidad de servicio del proceso de recolección domiciliario del Aseo Urbano explicada por las cuatro dimensiones.

\begin{tabular}{|c|c|c|c|c|c|c|c|c|c|}
\hline \multirow{2}{*}{ Componente } & \multicolumn{3}{|c|}{ Autovalores iniciales } & \multicolumn{3}{|c|}{$\begin{array}{l}\text { Sumas de las saturaciones al auadrado } \\
\text { de la extacción }\end{array}$} & \multicolumn{3}{|c|}{$\begin{array}{l}\text { Suma de las saturaciones al cuadrado de } \\
\text { la rotación }\end{array}$} \\
\hline & Total & $\begin{array}{l}\text { \% de la } \\
\text { varianza }\end{array}$ & $\begin{array}{c}\% \\
\text { acumulado }\end{array}$ & Total & $\begin{array}{l}\text { \% de la } \\
\text { varianza }\end{array}$ & $\begin{array}{c}\% \\
\text { acumulado }\end{array}$ & Total & $\begin{array}{l}\text { \% de la } \\
\text { varianza }\end{array}$ & $\begin{array}{c}\% \\
\text { acumulado }\end{array}$ \\
\hline 1 & 5,519 & 25897 & 25,807 & 5,419 & 25,807 & 25,807 & 3,0186 & 14,696 & 146996 \\
\hline 2 & 2,135 & 10,165 & 35,972 & 2,155 & vo, 165 & 36,972 & 2,994 & 14256 & 28,953 \\
\hline 3 & 1,996 & 9,506 & 45,478 & 1,996 & 9,506 & 45,478 & 2,761 & 13,149 & 42,102 \\
\hline 4 & 1853 & 89822 & 54,300 & 1,863 & 8,822 & 54,300 & 2,562 & 12,198 & 54,300 \\
\hline 5 & 1,541 & 7,338 & 61,638 & & & & & & \\
\hline 6 & 1,492 & 7,104 & 68,742 & & & & & & \\
\hline 7 & 1,127 & 5,369 & 74,111 & & & & & & \\
\hline $\mathbf{8}$ &, 875 & 4,168 & 78,279 & & & & & & \\
\hline 9 & ,827 & 3,938 & 82,217 & & & & & & \\
\hline 10 &, 675 & 3215 & 85,452 & & & & & & \\
\hline 11 &, $5 / 2$ & 2725 & 88, , 57 & & & & & & \\
\hline 12 &, 472 & 2750 & 90,406 & & & & & & \\
\hline 13 &, 396 & 1)885 & 92701 & & & & & & \\
\hline 14 &, 376 & 1,791 & 94,082 & & & & & & \\
\hline 15 & ,331 & 1,575 & 95,807 & & & & & & \\
\hline 16 & 249 & 1,184 & 96,841 & & & & & & \\
\hline 17 & 218 & 1,037 & 97,879 & & & & & & \\
\hline 18 & 159 & 759 & 98,637 & & & & & & \\
\hline 19 & , 126 & 599 & 99,237 & & & & & & \\
\hline 20 & , 10868 &, 418 & 99,655 & & & & & & \\
\hline 21 & ,073 &, 345 & 200,000 & & & & & & \\
\hline
\end{tabular}


Hair et al, (2008) presentan varios criterios para orientar en la decisión de cuándo considerar una carga factorial como significativa, una de ellas es la significación práctica que consiste en una regla totalmente empírica. Los autores indican que cargas factoriales mayores $a \pm 0.30$ se considera que están en el nivel mínimo; las cargas de \pm 0.40 se consideran más importantes, mientras que las cargas de \pm 0.50 o mayores, se consideran significativas. Explican además que cargas factoriales mayores a \pm 0.80 no son normales, en virtud de que el cuadrado de la carga factorial de una variable en un factor, representa la varianza total de la variable explicada por el factor.

Para realizar el análisis se revisan las cargas de los ítems para cada dimensión tomando como criterio aquellos items que cargan por encima de 0,42 en el factor. Al estudiar la cantidad de ítems que cargan en cada dimensión, resalta que en el primer factor cargan 17 de los 21 ítems, mientras que para los factores restantes cargan 4 ítems para el segundo y 3 ítems para la tercera y cuarta dimensión, se observa además como algunos de los ítems cargan en dos dimensiones. La interpretación de este resultado es muy compleja, por lo tanto se procede a realizar el análisis factorial con rotación varimax.

La matriz de componentes principales rotados, mostrada en la tabla 4, muestra los ítems que cargan o correlacionan en 0,3850 o más con cada factor, se observa un resultado en el cual la cantidad de ítems que carga por dimensión es mucho mas equitativa, pero cinco de los ítems cargan simultáneamente en dos o más factores.

A partir de este resultado se realiza el análisis usando el criterio de significación práctica propuesto por Hair et al,, (2008), contrastando además cada item con la fundamentación teórica de los factores.

Ya validada la estructura interna de la escala CASERAU, se procede a definir teóricamente las cuatro dimensiones que permiten medir la calidad de servicio del proceso de recolección domiciliario del aseo urbano en el municipio Maracaibo. Se asigna un nombre a cada dimensión y se describe su significado teórico.

\section{Factores que inciden en la calidad de servicio del proceso de recolección domiciliario en el Municipio Maracaibo.}

Como resultado del análisis factorial se establece que son cuatro los factores que afectan la calidad de servicio del proceso de recolección domiciliario en el municipio Maracaibo. Estos factores difieren de los propuestos inicialmente en la teoría a través del constructo, por lo tanto se hace necesario darles una nueva denominación y definirlos.

Factor 1: En esta primera dimensión o indicador, luego del análisis presentado anteriormente, quedan asignado los ítems: $13,16,18$ y 19 . Estos reactivos están referidos al proceso de comunicación entre usuarios del servicio y el prestador del mismo. Incluye aspectos que van desde los medios que se utilizan para comunicarse, así como la calidad de la información que se emite hacia los usuarios del servicio. Por lo tanto el primer factor queda denominado como:

Comunicación: esta referido a los aspectos dedicados al proceso de comunicación con los usuarios del servicio de recolección domiciliario, se 


\section{Tabla 4. \\ Matriz de componentes principales rotados para CASERAU}

\begin{tabular}{|c|cccc|}
\hline \multicolumn{5}{|c}{ Matriz de componentes rotados(a) } \\
\hline \multirow{2}{*}{ Ítem } & \multicolumn{4}{|c|}{ Componente } \\
\cline { 2 - 5 } & $\mathbf{1}$ & $\mathbf{2}$ & $\mathbf{3}$ & $\mathbf{4}$ \\
\hline 1 & 0,116 & 0,247 & 0,034 & 0,61 \\
2 & 0,179 & 0,211 & 0,435 & 0,17 \\
3 & 0,039 & 0,708 & 0,016 & 0,042 \\
4 & 0,249 & 0,341 & 0,151 & 0,579 \\
5 & 0,031 & 0,558 & 0,286 & 0,083 \\
6 & 0,082 & 0,511 & 0,445 & 0,219 \\
7 & 0,047 & 0,118 & 0,165 & 0,624 \\
8 & 0 & 0,096 & 0,405 & 0,679 \\
9 & 0,143 & 0,385 & 0,52 & 0,258 \\
10 & 0,361 & 0,292 & 0,668 & 0,156 \\
11 & 0,039 & 0,658 & 0,133 & 0,028 \\
12 & 0,312 & 0,659 & 0,164 & 0,26 \\
13 & 0,52 & 0,358 & 0,139 & 0,465 \\
14 & 0,172 & 0,238 & 0,767 & 0,167 \\
15 & 0,028 & 0,055 & 0,678 & 0,163 \\
16 & 0,717 & 0,34 & 0,168 & 0,033 \\
17 & 0,605 & 0,001 & 0,138 & 0,192 \\
18 & 0,728 & 0,08 & 0,209 & 0,286 \\
19 & 0,534 & 0,398 & 0,315 & 0,158 \\
20 & 0,464 & 0,225 & 0,189 & 0,57 \\
21 & 0,711 & 0,235 & 0,315 & 0,142 \\
\hline
\end{tabular}

Método de extracción: Análisis de componentes principales.

Método de rotación: Normalización Varimax con Kaiser.

a. La rotación ha convergido en 8 iteraciones.

consideran: material informativo, medios de comunicación utilizados, la frecuencia y calidad de los mismos.

Factor 2: Los ítems que explican a este factor son: 3, 5, 6, 11 y 12, al leerlos se aprecia que todos convergen en la habilidad que tiene el instituto que presta el servicio, a través de sus trabajadores, para dar respuesta certera y oportuna a los requerimientos de los usuarios.

La capacidad de atender rápidamente los problemas planteados por los usuarios, la existencia de personal y medios para colocar reclamos, la disposición de los trabajadores para atender los reclamos y la capacidad de conocer las necesidades reales de los usuarios del servicio de recolección, son aspectos que inciden en la calidad de servicio del proceso de recolección domiciliario. La denominación para este factor se muestra a continuación: 
Calidad de servicio en el proceso de recolección domiciliario de residuos sólidos Sáez Molero, Alejandrina Luz y Urdaneta Graterol, Joheni Alejandra

Capacidad de Respuesta: Capacidad del instituto que presta el servicio para atender oportunamente los requerimientos de los usuarios del servicio de recolección.

Factor 3. Como resultado del análisis factorial y la revisión del constructo teórico en el tercer factor se asignan los ítems: 2, 9, 10, 14, 17 y 20, reactivos referidos, en su totalidad, a la frecuencia y horarios de recolección. Se consideran elementos, que debe cumplir el instituto que presta el servicio de recolección de residuos sólidos, tales como: la frecuencia con que se recolectan los residuos sólidos, los días de la semana fijados para la recolección, la notificación de los horarios de recolección y sus modificaciones, así como la conveniencia para los usuarios de los horarios en los que se presta el servicio.

Frecuencia y horario de recolección: Confianza en que el servicio de recolección domiciliario se presta con la frecuencia y en el horario prometido.
Factor 4: Los ítems asigandos en este factor son: 1, 4, 7, 8 ,15 y 21, los cuales se refieren a los elementos que son palpables durante el proceso de recolección de residuos sólidos.

Características como las condiciones del camión utilizado para la recolección, la apariencia de los trabajadores que ejecutan el proceso de recolección, así como el trato que dan y las condiciones de los dispositivos de almacenamiento de residuos sólidos utilizados en el origen, son consideradas influyentes en la calidad del servicio. El ítem referido a las transacciones se enmarca en los aspectos tangibles de las mismas. El nombre designado al factor se presenta a continuación:

Elementos Tangibles: Apariencia de los equipos, personal y material de comunicación referentes al proceso de recolección.

La tabla 5 resume los factores con sus confiabilidades y la confiabilidad del instrumento.

Tabla 5.

Confiabilidad para la escala y los factores que miden la calidad de servicio del proceso de recolección de residuos sólidos del Municipio Maracaibo

\begin{tabular}{lc}
\hline \multicolumn{1}{c}{ Factor } & Confiabilidad \\
\hline Comunicación & 0,706 \\
Capacidad de respuesta & 0,711 \\
Frecuencia y horario de recolección & 0,731 \\
Elementos tangibles & 0,611 \\
\hline \multicolumn{2}{c}{ Instrumento } \\
\hline
\end{tabular}


Las dimensiones resultantes no son idénticas a las propuestas por Zeithaml et al, (1993). Debido a las características del servicio estudiado, el factor más importante lo constituye la frecuencia y horario de recolección por ser un aspecto clave en la prestación del servicio; los factores capacidad de respuesta y elementos tangibles se mantienen del instrumento tomado como base en la investigación enfatizando que son aspectos relevantes para el servicio publico de recolección domiciliario de residuos sólidos; por su parte la dimensión comunicación, resulta también un factor importante para este servicio público, solo que dicha dimensión fue presentada en las versiones iniciales de los estudios realizados por Zeithaml et al, (1993), mas no aparece como dimensión en la adaptación final del instrumento diseñado por los autores.

A este nivel del estudio se han completado todos los pasos y análisis que se requieren para establecer la validez interna de un instrumento documental, por ende para la escala diseñada y constituída por 21 ítems, denominada CASERAU, queda demostrada su validez interna. Se presenta a continuación los reactivos del instrumento resultante en la Tabla 6 y se da inicio entonces a la aplicación de las técnicas necesarias para determinar si CASERAU posee validez externa.

\subsubsection{Validez de criterio}

Una de las propiedades que debe tener un instrumento documental es la de validez de criterio, esta consiste en que los resultados obtenidos con el instrumento diseñado deben ser consistentes con los resultados de otro instrumento validado, al aplicar ambos en una misma población (Arribas Martín, 2004; Prieto y Delgado, 2010).

\section{Tabla 6}

Ítems de CASERAU

\begin{tabular}{|c|c|c|c|c|c|}
\hline DECLARACIONES (ATRIBUTOS) & & te en des- & $\square$ & $\begin{array}{l}\text { Fuertemente } \\
\text { acuerdo }\end{array}$ & de \\
\hline $\begin{array}{l}\text { 1. EI IMAU carece de atención personalizada } \\
\text { para los usuarios. }\end{array}$ & 1 & 2 & 3 & 4 & \\
\hline $\begin{array}{l}\text { 2. Existe diversidad de medios para comuni- } \\
\text { carse (números de teléfonos, buzón de quejas, } \\
\text { etc.) con el IMAU. }\end{array}$ & 1 & 2 & 3 & 4 & \\
\hline $\begin{array}{l}\text { 3. Los folletos informativos emitidos por el } \\
\text { IMAU, para el usuario, son atractivos. }\end{array}$ & 1 & 2 & 3 & 4 & \\
\hline $\begin{array}{l}\text { 4. Los trabajadores que recogen los desechos } \\
\text { sólidos carecen de conocimientos para respon- } \\
\text { der sus inquietudes respecto al servicio. }\end{array}$ & 1 & 2 & 3 & 4 & \\
\hline $\begin{array}{l}\text { 5. EI IMAU resuelve de manera rápida los pro- } \\
\text { blemas que usted plantea }\end{array}$ & 1 & 2 & 3 & 4 & \\
\hline $\begin{array}{l}\text { 6. El IMAU carece de canales de comunicación } \\
\text { con el usuario para la formulación de reclamos. }\end{array}$ & 1 & 2 & 3 & 4 & \\
\hline $\begin{array}{l}\text { 7. EI IMAU cuenta con empleados para aten- } \\
\text { der reclamos. }\end{array}$ & 1 & 2 & 3 & 4 & \\
\hline
\end{tabular}




\section{Cont... Tabla 6}

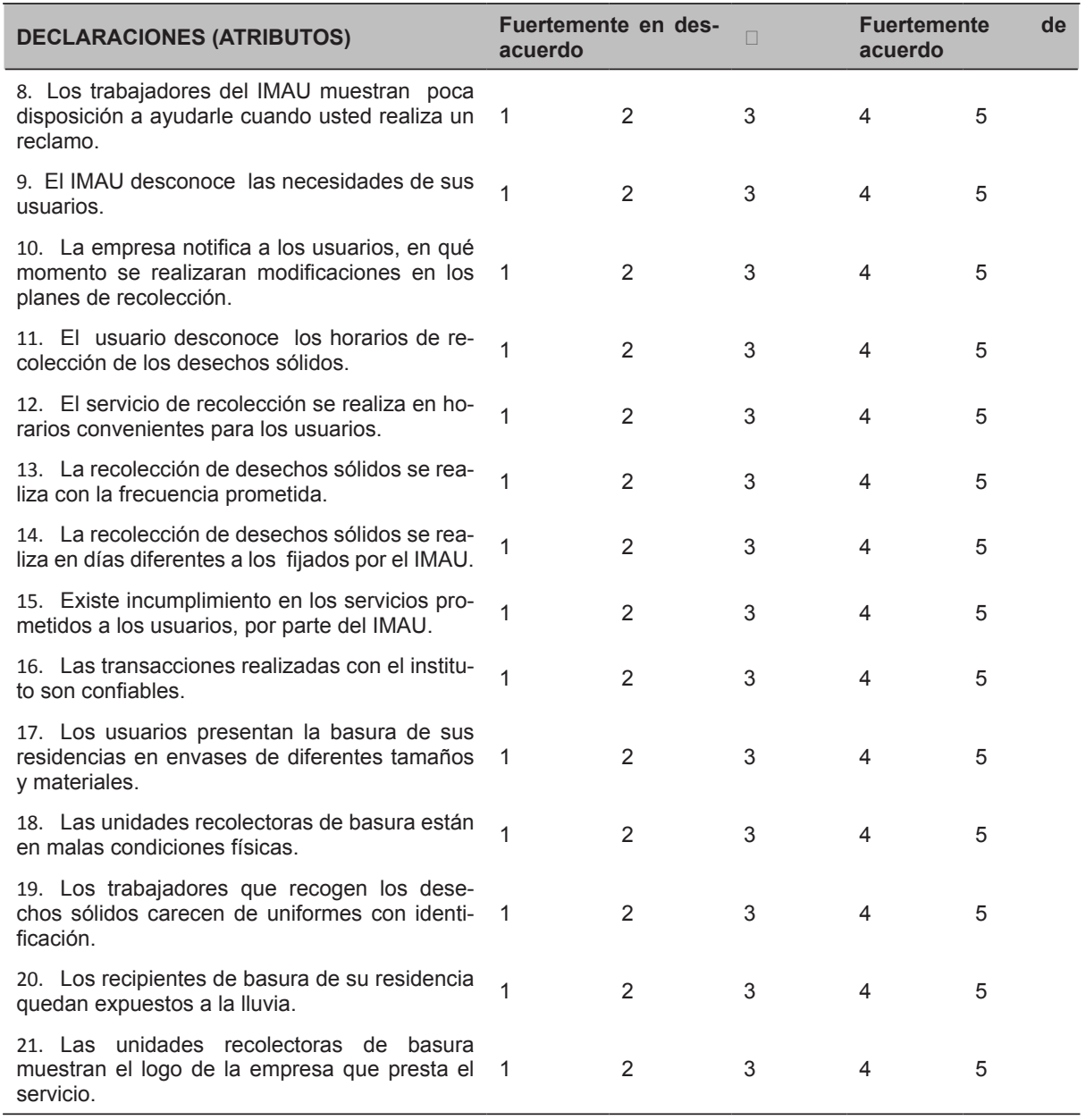

Fuente: elaboracion propia

En el caso del presente estudio se puede indicar que se aplica validez concurrente, en virtud de que se cuenta con un instrumento validado para medir la calidad de servicio y se toman los datos simultaneamente con CASERAU y el instrumento patrón. El instrumento que se toma como patrón es el SERVPEF de Cronin y Taylor (1992).

Tanto para CASERAU como para el instrumento patrón el valor final de medición de la variable es númerico, lo que permite la utilización del coeficiente de correlación de pearson para tomar 
una decisión respecto a la veracidad de la hipótesis nula de no existencia de validez de criterio.

Para la realización de esta prueba se aplican ambos instrumentos, CASERAU y el patrón, a un total de 38 sujetos. Una vez recolectados los datos, con la utilización del PASW.18 se verifica la existencia de relación lineal entre la CalidadC medida con CASERAU y la CalidadP medida con el instrumento patrón, en un gráfico de dispersión (gráfico 1).
El gráfico sugiere la existencia de asociación lineal entre la calidad de servicio medida con el instrumento patrón y la calidad de servicio medida con CASERAU, ambas mediciones se asocian de forma directamente proporcional.

Seguidamente se realiza la prueba de hipótesis para demostrar que el coeficiente de correlación $(\rho)$ entre ambas medidas es igual a cero, lo que señala la no existencia de correlación entre ambas mediciones.

\section{Gráfico 1. Diagrama de dispersión de CalidadC vs CalidadP}

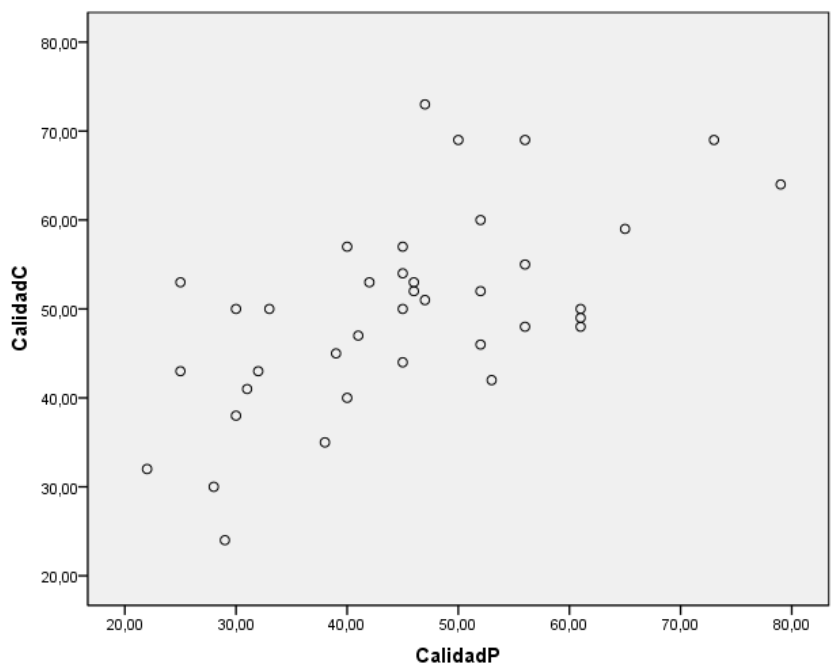

Fuente: elaboración propia

El resultado de la prueba indica que se rechaza la hipótesis de no existencia de validez de criterio, al obtenerse un Valor P igual a 0,000, el cual es menor al nivel de significancia de 0,01 . Estos resultados se muestran en la tabla 7 , en la cual también se observa que el coeficiente de correlación de pearson estimado $(R)$ es de 0,614 , lo que lleva a concluir que la CalidadC estimada con CASERAU se asocia con una recta de pendiente positiva con la CalidadP estimada con el patrón, y esta correlación entre las dos variables 
Calidad de servicio en el proceso de recolección domiciliario de residuos sólidos

Sáez Molero, Alejandrina Luz y Urdaneta Graterol, Joheni Alejandra

es considerada como moderada según Aguayo y Lora (2014). Se verifica entonces, que CASERAU posee validez de criterio concurrente.

Tabla 7

\section{Resultados de la prueba de hipótesis para verificar la existencia de validez de criterio}

\begin{tabular}{llcc}
\hline \multicolumn{3}{c}{ Correlaciones } \\
\hline \multirow{3}{*}{ CalidadC CalidadC } & CalidadP \\
& Correlación de Pearson & 1 & $0,614^{* *}$ \\
& Sig. (bilateral) & 38 & 0,00 \\
& $N$ & 38 \\
\hline CalidadP & Correlación de Pearson & $0,614^{* *}$ & 1 \\
& Sig. (bilateral) & 0,00 & \\
& $N$ & 38 & 38 \\
\hline
\end{tabular}

${ }^{\star *}$. La correlación es significativa al nivel 0,01 (bilateral).

Fuente: elaboración propia

\subsubsection{Reproducibilidad}

Esta propiedad permite medir desde afuera la calidad del instrumento diseñado, consiste en aplicar la escala a los mismos sujetos por dos o mas evaluadores, con la finalidad de verificar la hipótesis nula de existencia de reproducibilidad del instrumento entre encuestadores. La pueba, para CASERAU, está basada en un modelo lineal univariante donde los sujetos y los encuestadores son los factores que afectan a la variable objeto de estudio. EI rechazo de la hipótesis nula que plantea la no incidencia de los encuestadores sobre el resultado (calidad) nos llevaría a concluir la no reproducibilidad de CASERAU; mientras que el rechazo de la hipótesis nula que plantea la no diferencia de las mediciones entre los sujetos nos llevaría a concluir la existencia de discriminación por parte del instrumento.
La verificación de la propiedad de reproducibilidad se realiza aplicando a 13 sujetos la escala diseñada CASERAU, por parte de dos encuestadores. Los resultados obtenidos se presentan en la tabla 8 , apreciándose un Valor $\mathrm{P}=0,086$ para el factor Encuestador, lo que lleva a decidir para un nivel de significancia de 0,05 que no puede rechazarse la hipótesis nula, concluyéndose que no existen diferencias significativas entre los resultados obtenidos por los dos encuestadores. Con este resultado se verifica que CASERAU es una escala que cumple con el criterio de reproducibilidad.

Respecto a la prueba que compara la media de Calidad entre sujetos, se obtiene que existe diferencia significativa entre las mediciones de cada sujeto (Valor $\mathrm{P}=0$ ) esto permite validar que el instrumento discrimina las percepciones de los usuarios. 
Tabla 8.

\section{Resultados de la prueba de hipótesis para verificar la existencia de estabilidad para CASERAU}

\begin{tabular}{|c|c|c|c|c|c|}
\hline \multicolumn{6}{|c|}{ Pruebas de los efectos inter-sujetos } \\
\hline Origen & $\begin{array}{c}\text { Suma de } \\
\text { cuadrados } \\
\text { tipo III }\end{array}$ & gl & $\begin{array}{c}\text { Media } \\
\text { cuadrática }\end{array}$ & $\mathbf{F}$ & Sig. \\
\hline Modelo & $77511,385^{a}$ & 14 & 5536,527 & 493,542 & 0 \\
\hline Encuestador & 39,385 & 1 & 39,385 & 3,511 & 0,086 \\
\hline Sujeto & 1439,846 & 12 & 119,987 & 10,696 & 0 \\
\hline Error & 134,615 & 12 & 11,218 & & \\
\hline Total & 77646 & 26 & & & \\
\hline
\end{tabular}

a. $\mathrm{R}$ cuadrado $=, 998$ ( $\mathrm{R}$ cuadrado corregida $=, 996)$

Fuente: elaboración propia

\subsection{Rendimiento del instrumento}

En esta etapa del estudio se tiene como objetivo optimizar la escala diseñada, de tal manera que se minimicen los errores al momento de tomar decisiones sobre la calidad de servicio percibida por los usuarios del proceso de recolección domiciliario. Es importante establecer para que valor de la variable debe calificarse el servicio evaluado como que no tiene calidad, para esto se utiliza la curva ROC (Receiver Operating Characteristic) herramienta estadística que constituye una representación gráfica de la eficacia de un clasificador, proporcionando un instrumento visual para examinar la relación entre la capacidad del clasificador para detectar correctamente los individuos con presencia de la condición de interés y su incapacidad para identificar los individuos del grupo de ausencia (Franco y Vivo, 2007).

Para establecer el rendimiento de CASERAU se toman los datos recolectados para la prueba de validez de criterio, dicotomizando los resultados obtenidos tanto con CASERAU como con el instrumento patrón. Es importante recordar que el instrumento patrón diseñado por Cronin y Taylor (1992) para medir la calidad de un servicio a traves de la percepción que tienen los usuarios de este, cuenta con 22 ítems.

Se requiere, previa a la aplicación de la curva ROC, establecer dos categorias para la calidad de servicio medida con el instrumento patrón, una para calificar que el servicio tiene calidad y otra que la complemente estableciendo la ausencia de calidad. García y Tejedor (2011) afirman que si la variable medida es politómica se opta por la agrupación dicotómica que mas interese al investigador, en virtud de esto y debido a que la mayoria de los sujetos 
Calidad de servicio en el proceso de recolección domiciliario de residuos sólidos Sáez Molero, Alejandrina Luz y Urdaneta Graterol, Joheni Alejandra

encuestados califican el servicio con los valores 1 y 2 , es evidente que los anchos para cada clase no son iguales, por lo que se decide que para los valores de 1 y 2 se califica al servicio como que no tiene calidad; mientras que para las puntuaciones que van del 3 al 5 , se considera que el servicio posee calidad.

Con la ayuda del PASW.18 se diseña la curva ROC para un total de 38 sujetos encuestados, resultado que se presenta en la Gráfica 2. Una medida de la calidad del clasificador está dada por el área bajo la curva, si el área es 1 el clasificador es ideal, un área de 0 señala una exactitud nula y un resultado de 0,5 (sobre la diagonal pricipal) indica que el clasificador es aleatorio (García y Tejedor, 2011). La curva obtenida queda por encima de la diagonal principal, con un porcentaje de área de que sugiere que el instrumento es útil.

\section{Tabla 9. \\ Rendimiento de CASERAU}

Área bajo la curva

Variables resultado de contraste:CalidadInstrumento

\begin{tabular}{|c|c|c|c|c|}
\hline \multirow[b]{2}{*}{ Área } & \multirow[b]{2}{*}{ Error típ. ${ }^{a}$} & \multirow[b]{2}{*}{ Sig. asintóticab } & \multicolumn{2}{|c|}{$\begin{array}{c}\text { Intervalo de confianza } \\
\text { asintótico al } 95 \%\end{array}$} \\
\hline & & & Límite inferior & Límite superior \\
\hline ,834 & ,067 & 001 & ,703 & ,964 \\
\hline
\end{tabular}

a. Bajo el supuesto no paramétrico

b. Hipótesis nula: área verdadera $=0,5$

Fuente: elaboración propia

El área resultante bajo la curva es de 0,834 tal como se presenta en la tabla 9. Esto indica que existe una probabilidad del $83,4 \%$ de que la calificación al servicio de recolección cuando este tiene calidad sea correcta, se tiene entonces que la capacidad discriminatoria del instrumento es buena.

Para la determinación del conjunto de valores de la variable para los cuales se calificará al servicio de recolección domiciliario como que tiene calidad, se ubica el punto de corte óptimo en la curva ROC. En la gráfica 2 se muestra que dicho punto proporciona una sensibilidad de 0,864 y una especificidad de 0,687; par ordenado que se ubica en la tabla de coordenadas de la curva generada por el PASW.18 observándose que está asociado con un valor de suma igual a 47,5 . 


\section{Gráfico 2.}

Curva ROC y el punto de corte óptimo

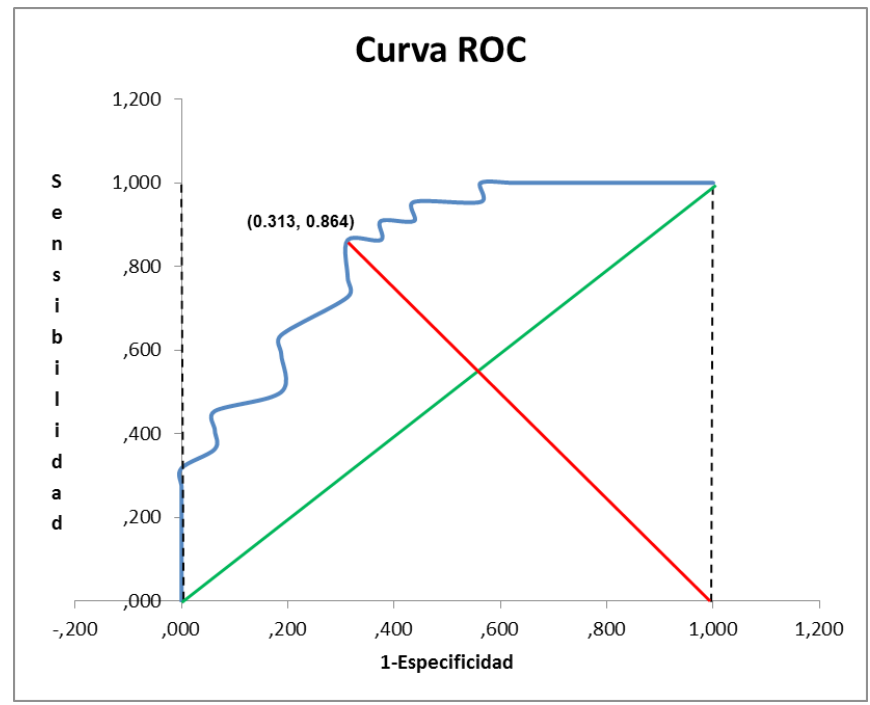

Fuente: elaboración propia

Tabla 10.

Dicotomización de la variable calidad de servicio para CASERAU

\begin{tabular}{llll} 
VALOR FINAL & SUMA & NIVEL DE CALIDAD & INTERPRETACIÓN \\
\hline$[1,2.29]$ & {$[21,48]$} & No tiene calidad & $\begin{array}{l}\text { Las expectativas del usuario no son } \\
\text { cumplidas, por lo tanto percibe la cali- } \\
\text { dad de servicio como inaceptable. }\end{array}$ \\
\hline$(2.29,5]$ & $(48,105]$ & Tiene calidad & $\begin{array}{l}\text { Las expectativas son cumplidas, por } \\
\text { lo tanto percibe la calidad de servicio } \\
\text { como aceptable.. }\end{array}$ \\
\hline
\end{tabular}

Fuente: elaboración propia

Se tiene entonces que para valores de suma (media) mayores a $48(2,29)$ se indicará que el servicio de recolección domiciliario posee calidad, mientras que para valores menores o iguales a $48(2,29)$ se calificará al servicio de que no tiene calidad. En la tabla 10 , se presentan los valores finales medidos y los valores de suma para los cuales se considera que existe calidad en el servicio de recolección. 
Calidad de servicio en el proceso de recolección domiciliario de residuos sólidos

Sáez Molero, Alejandrina Luz y Urdaneta Graterol, Joheni Alejandra

\section{Conclusiones}

El instrumento diseñado y denominado CASERAU resultó ser altamente confiable al obtener un Alpha de Cronbach de 0,845; su validez de contenido está respaldada por el juicio de tres expertos, mientras que la validez de constructo se sustenta con la técnica de análisis factorial.

Como resultado del AF se obtuvieron cuatro dimensiones, con confiabilidades entre altas y buenas, adaptadas a la manera en que debe valorarse la calidad de servicio del proceso de recolección domiciliario según la percepción del usuario marabino; los factores establecidos fueron: comunicación, capacidad de respuesta, frecuencia y horario de recolección y aspectos tangibles. Se obtienen dos dimensiones que están presentes en el modelo teórico inicial y dos dimensiones específicas al servicio estudiado.

CASERAU presenta una validez de criterio concurrente significativa al observar que sus mediciones tienen correlación positiva de 0,614 con las medidas del instrumento patrón utilizado. El instrumento resultante es confiable y posee validez de contenido, de constructo y de criterio.

Los resultados presentados para la curva ROC demuestran que el instrumento es discriminante ya que en $83,4 \%$ de las veces que se utilice para evaluar la calidad del servicio la medición será correcta. El instrumento tiene una sensibilidad de 0,864 es decir que $86,4 \%$ de las veces que se califique que el servicio tiene calidad se estará en lo correcto; mientras que la especificidad es del 0,687 , lo que indica que el $68,7 \%$ de las veces que se califique al servicio sin calidad, se hará correctamente.
El instrumento tiene un excelente rendimiento.

Para finalizar se tiene que en el municipio Maracaibo y para la población objeto de estudio, una puntuación promedio de la percepción de los usuarios de mas de 2,29 (equivalente a una suma de 48 puntos) permite calificar que el servicio de recolección presenta calidad; mientras que valores obtenidos por debajo o iguales al punto de corte mostrado, llevan a calificar al servicio como que no presenta calidad.

\section{Referencias Bibliográficas}

Aguayo, Mariano., y Lora, Estrella. (2014). Fabis: Fundación andaluza Beturia para la Investigación en Salud. Retrieved from Fabis Organización: http://www.fabis.org/html/ archivos/docuweb/contraste_hipotesis_3r.pdf

Arribas, Martín. (2004). Diseño y validación de cuestionarios. Matronas Profesion, 23-29.

Bigné, José., Moliner, Miguel., Vallet, Teresa., y Sánchéz, Javier. (1999). Un estudio comparativo de los instrumentos de medición de la calidad de los servicios públicos. Castellon: Universitat jaume I.

Blanco, Neligia. (2000). Instrumentos de Recolección de Datos Primarios. Maracaibo: Departamento de Artes Plásticas.Dirección de Cultura. LUZ.

Carvajal, Ana, Centeno, Carlos., Watson, Roger, Martínez, Marina, y Sanz, Alvaro (2011). ¿Cómo validar un instrumento de medida de la salud? An.Sist.Sanit. Navar, 63-72.

Ceballos Méndez, Edward Jonnathan. (2009, Marzo 28). Saber ULA. Retrieved from Saber ULA: http://www.saber.ula.ve/bits- 
tream/123456789/28629/1/articulo3. pdf

Duque Oliva, Edison (2005). Revisión del concepto de calidad del servicio y sus modelos de medición. INNOVAR, revista de ciencias administrativas y sociales, 64-80.

Franco, Manuel, y Vivo, Juana María (2007). Análisis de curvas ROC. Principios básicos y aplicaciones. Madrid: La Muralla.

García, Ana, y Tejedor, Francisco. (2011). Variables TIC vinculadas a la generación de nuevos escenarios de aprendizaje en la enseñanza universitaria. Aportes de las curvas ROC para el análisis de diferencias. Educación XX, 43-78.

García, José Antonio, Reading, Arturo, y López, Juan Carlos (2013). Cálculo del tamaño de la muestra en investigación en educación médica. Investigación en Educación Médica, 217-224.

Hair, Joseph, Anderson, Rolph, y Tatham, Ronald y Black, William (2008). Análisis Multivariante. España: Pearson. Prentice Hall.

Hernández, Roberto, Fernández, Carlos, y Baptista, Pilar (2006). Metodología de la investigación. México DF: Mc Graw Hill Interamericana.

Lagunes, Roberto (2017). Recomendaciones sobre los procedimientos de construcción y validación de instrumentos y escalas de medición en la psicología de la salud. Psicología y
Salud, 5-18.

Levy, Jean Pierre, y Varela, Jesús (2003). Análisis Multivariante para las Ciencias Sociales. Madrid: Pearson Educación S.A.

Maldonado, Sonia, Guillén, Ana, y Carranza, Rafael (2013). Factores determinantes de la calidad del servicio de una cafetería en el campus de una universidad pública. Revista internacional administración y finanzas, 109-118.

Monje Álvarez, Carlos Arturo (2011). Metodología de la Investigación. Cuantitativa y cualitativa. Guía didáctica. Neiva: Universidad SurColombiana.

Montaña, Joaquin, Ramirez, Elias, y Ramirez, Hernando (2002). Evaluación de la calidad de los servicios públicos domiciliarios. Revista Colombiana de Marketing, 47-62.

Paniagua, Ramón (2015). Metodología para la validación de una escala o instrumento de medida. Medellin, Colombia: Facultad Nacional de Salud Pública.

Prieto, Gerardo, y Delgado, Ana (2010). Fiabilidad y validez. Papeles del Psicólogo, 67-74.

Sierra, Restituto (2000). Técnicas de Investigación Social. Teoría y Ejercicios. Madrid: Paraninfo.

Zeithaml, Valarie, Parasuraman, A., y Berry, Leonard (1993). Calidad total en la gestión de servicios. Madrid: Ediciones Díaz de Santos, S.A.

- Esta obra está bajo una licencia de Creative Commons Reconocimiento-NoComercial-

Compartirlgual 3.0 Unported.

http://creativecommons.org/licenses/by-nc-sa/3.0/deed.es_ES 